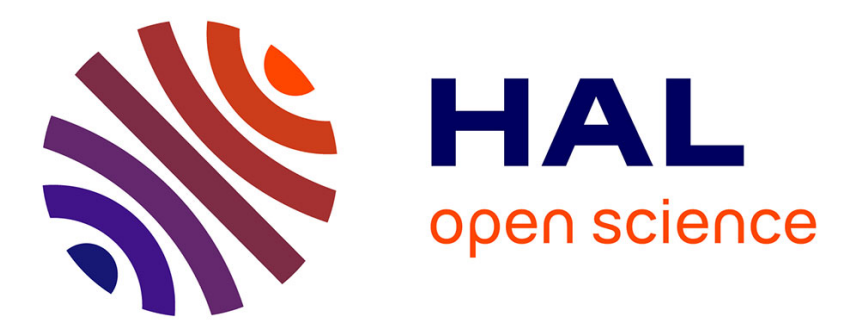

\title{
Rotational forms of Large Eddy Simulation turbulence models: modeling and mathematical theory
}

\author{
Luigi C. Berselli, Roger Lewandowski, Dinh Duong Nguyen
}

\section{To cite this version:}

Luigi C. Berselli, Roger Lewandowski, Dinh Duong Nguyen. Rotational forms of Large Eddy Simulation turbulence models: modeling and mathematical theory. Chinese Annals of Mathematics - Series B, 2021, 42 (1), pp.1-24. 10.1007/s11401-021-0243-z . hal-02569244

\section{HAL Id: hal-02569244 \\ https://hal.science/hal-02569244}

Submitted on 11 May 2020

HAL is a multi-disciplinary open access archive for the deposit and dissemination of scientific research documents, whether they are published or not. The documents may come from teaching and research institutions in France or abroad, or from public or private research centers.
L'archive ouverte pluridisciplinaire HAL, est destinée au dépôt et à la diffusion de documents scientifiques de niveau recherche, publiés ou non, émanant des établissements d'enseignement et de recherche français ou étrangers, des laboratoires publics ou privés. 


\title{
Rotational forms of Large Eddy Simulation turbulence models: modeling and mathematical theory
}

\author{
Luigi C. Berselli ${ }^{1}$, Roger Lewandowski ${ }^{2}$, and Dinh Duong Nguyen ${ }^{3}$ \\ ${ }^{1}$ Università di Pisa, Dipartimento di Matematica, Via Buonarroti 1/c, I-56127 \\ Pisa, Italy, E-mail: luigi.carlo.berselli@unipi.it \\ ${ }^{2,3}$ IRMAR, UMR CNRS 6625, University of Rennes 1 and FLUMINANCE Team, \\ INRIA Rennes, France, E-mail: Roger.Lewandowski@univ-rennes1.fr, \\ dinh-duong.nguyen@univ-rennes1.fr
}

May 11, 2020

\begin{abstract}
In this paper we present a derivation of a back-scatter rotational Large Eddy Simulation model, which is the extension of the Baldwin and Lomax model to nonequilibrium problems. The model is particularly designed to mathematically describe a fluid filling a domain with solid walls and consequently the differential operators appearing in the smoothing terms are degenerate at the boundary. After the derivation of the model, we prove some of the mathematical properties coming from the weighted energy estimates and which allow to prove existence and uniqueness of a class of regular weak solutions.
\end{abstract}

Key words: Fluid mechanics; Turbulence models; Rotational Large Eddy Simulation models; Navier-Stokes Equations.

2010 MSC: 76D05, 35Q30, 76F65, 76D03, 35Q35.

\section{Introduction}

The aim of this paper is twofold: From one side we are deriving in a consistent way a rotational Large Eddy Simulation model, capable of taking into account of back-scatter of energy; from another side we are also showing, by using rather elaborate functional analysis tools, the existence of weak solutions for the models we propose.

Recall that, the motion of a turbulent incompressible flow in a $3 \mathrm{D}$ domain $\Omega$ can be simulated by using a turbulence model such as the following eddy viscosity model $^{1}$ :

$$
\left\{\begin{array}{r}
\overline{\mathbf{v}}_{t}+\operatorname{div}(\overline{\mathbf{v}} \otimes \overline{\mathbf{v}})-\operatorname{div}\left(\left(2 \nu+\nu_{\text {turb }}\right) \mathbf{D} \overline{\mathbf{v}}\right)+\nabla \bar{p}=\mathbf{f}, \\
\operatorname{div} \overline{\mathbf{v}}=0,
\end{array}\right.
$$

\footnotetext{
${ }^{1}$ Thanks to the divergence free constraint $\operatorname{div} \overline{\mathbf{v}}=0$, we have $\operatorname{div}(\overline{\mathbf{v}} \otimes \overline{\mathbf{v}})=(\overline{\mathbf{v}} \cdot \nabla) \overline{\mathbf{v}}$ where $\overline{\mathbf{v}} \otimes \overline{\mathbf{v}}=\left(\bar{v}_{i} \bar{v}_{j}\right)$ for $1 \leq i, j \leq 3$. Therefore, these both forms are used throughout this report without any confusion, but also the "rotational form" will be used.
} 
where $\overline{\mathbf{v}}_{t}=\partial_{t} \overline{\mathbf{v}}$ for simplicity, $\overline{\mathbf{v}}=\overline{\mathbf{v}}(t, \mathbf{x})=\left(\bar{v}_{1}(t, \mathbf{x}), \bar{v}_{2}(t, \mathbf{x}), \bar{v}_{3}(t, \mathbf{x})\right)$ is the mean velocity of the fluid, $p=\bar{p}(t, \mathbf{x})$ the mean pressure, $\nu>0$ the kinematic viscosity, $\nu_{\text {turb }} \geq 0$ the eddy viscosity (also known as the turbulent viscosity), $\mathbf{f}=\mathbf{f}(t, \mathbf{x})=\left(f_{1}(t, \mathbf{x}), f_{2}(t, \mathbf{x}), f_{3}(t, \mathbf{x})\right)$ the external source term, $\mathbf{D} \overline{\mathbf{v}}=\frac{1}{2}\left(\nabla \overline{\mathbf{v}}+\nabla \overline{\mathbf{v}}^{t}\right)$ the deformation stress of the mean velocity, and "div" stands for the divergence operator.

In the whole paper we will consider the problem with homogeneous Dirichlet boundary conditions, i.e.,

$$
\overline{\mathbf{v}}=\mathbf{0} \quad \text { on } \quad(0, T) \times \partial \Omega,
$$

and this poses certain technical problems, which are not present in the case of homogeneous turbulence treated in the whole space or in the space-periodic setting. However, numerical simulations would require the use of wall laws (see [7]).

One basic problem in turbulence modeling is the determination of the eddy viscosity $\nu_{\text {turb }}$, for which there are many options (see a comprehensive presentation of this question in $[4,7]$ ). One of the most popular models (and one among the first introduced) is the Smagorinsky one [19] for which the eddy viscosity is given by

$$
\nu_{\text {turb }}=\kappa \ell^{2}|\mathbf{D} \overline{\mathbf{v}}|,
$$

where $\kappa$ is the von Kármán dimensionless constant (the value of which is about 0.41 ) and $\ell$ is the Prandtl mixing length (see [17]). The peculiarity of the modeling and of the equations derived is the degeneracy of the differential operators (by means of the function $\ell(\mathbf{x})$, which is vanishing at the boundary). The models we study can be interpreted as obtained with the application of a differential filter with radius vanishing near to the boundary; hence, the model is not over-smoothing the boundary layer. The analysis of wall-laws or of other boundary conditions requires tools not developed yet for this problem.

In the case of a flow over a plate, which is identified with the plane $(x, y, 0)$, then the domain is $\Omega=\mathbb{R}^{2} \times\{z>0\}$ and one finds in Obukhov [16] the following law

$$
\ell=\ell(z)=\kappa z .
$$

Considering a bi-layer model for a turbulent boundary layer over a plate, Baldwin \& Lomax [2] suggested -from heuristic arguments- to use in the inner part of boundary layer the following formula

$$
\nu_{\text {turb }}=\kappa \ell^{2}(z)|\bar{\omega}|
$$

where $\overline{\boldsymbol{\omega}}=\operatorname{curl} \overline{\mathbf{v}}$ denotes the mean vorticity, while the function $\ell$ (not a constant now) is determined by the Van Driest formula [20],

$$
\ell(z):=\kappa z\left(1-\mathrm{e}^{-z / A}\right)
$$

here $A$ depends on the oscillations of the plate and on the kinematic viscosity $\nu$, while $z \geq 0$ is again the distance from the plate. As it is well-known, the Smagorinsky model is over-diffusive, and model (1.2) looks to be a very interesting alternative, leading by (1.1) to the system

$$
\left\{\begin{array}{r}
\overline{\mathbf{v}}_{t}+\operatorname{div}(\overline{\mathbf{v}} \otimes \overline{\mathbf{v}})-\nu \Delta \overline{\mathbf{v}}-\operatorname{div}\left(\kappa \ell^{2}(z)|\overline{\boldsymbol{\omega}}| \mathbf{D} \overline{\mathbf{v}}\right)+\nabla \bar{p}=\mathbf{f} \\
\operatorname{div} \overline{\mathbf{v}}=0
\end{array}\right.
$$


However, the eddy viscosity term $-\operatorname{div}\left(\kappa \ell^{2}(z)|\overline{\boldsymbol{\omega}}| \mathbf{D} \overline{\mathbf{v}}\right)$ in (1.3) does not follow the rotational structure of formula (1.2). In [18], the authors suggest a purely rotational form $\operatorname{curl}\left(\kappa \ell^{2}(\mathbf{x})|\overline{\boldsymbol{\omega}}| \overline{\boldsymbol{\omega}}\right)-$ which is consistent with (1.2)- yielding the following system

$$
\left\{\begin{array}{r}
\overline{\mathbf{v}}_{t}+\operatorname{div}(\overline{\mathbf{v}} \otimes \overline{\mathbf{v}})-\nu \Delta \overline{\mathbf{v}}+\operatorname{curl}\left(\kappa \ell^{2}(\mathbf{x})|\overline{\boldsymbol{\omega}}| \overline{\boldsymbol{\omega}}\right)+\nabla \pi=\mathbf{f}, \\
\operatorname{div} \overline{\mathbf{v}}=0,
\end{array}\right.
$$

for some modified pressure term $\pi$.

In addition to being over-diffusive, the Smagorinsky model (but this limitation is also shared by non adaptive eddy viscosity models) is not capable of taking into account phenomena of back-scatter of energy. Consequently, system (1.4) seems of interest limited to (statistically) stationary or equilibrium flows. A first complete existence theory for the Baldwin \& Lomax model in the steady case has been recently given in [3].

In order to consider more complex physical settings, a variant has been proposed in [18] including a non-smoothing dispersive term, in the same spirit as in Voigt models (also written as Voight sometimes). The mathematical theory in this case needs to handle degenerate operators and weighted estimates. For this reason, in [1] we have modeled a back-scatter term of a Voigt form such as $-\alpha \operatorname{div}\left(\ell(\mathbf{x}) \mathbf{D} \overline{\mathbf{v}}_{t}\right)$, where $\alpha>0$ denotes the length scale, for turbulence evolving towards a statistical equilibrium, where $\ell(\mathbf{x})$ is a smooth positive function, vanishing only at the boundary of the domain and with a prescribed rate. In [1] we also studied the properties of the corresponding PDE system, in conjunction with TKE (turbulent kinetic energy equations). In [18], the authors suggested instead a back-scatter term under rotational form, such as curl $\left(\ell^{2}(\mathbf{x}) \overline{\boldsymbol{\omega}}_{t}\right)$, obtaining the following system:

$$
\left\{\begin{array}{r}
\overline{\mathbf{v}}_{t}+\operatorname{curl}\left(\ell^{2}(\mathbf{x}) \overline{\boldsymbol{\omega}}_{t}\right)+\operatorname{div}(\overline{\mathbf{v}} \otimes \overline{\mathbf{v}})-\nu \Delta \overline{\mathbf{v}}+\operatorname{curl}\left(\kappa \ell^{2}(\mathbf{x})|\overline{\boldsymbol{\omega}}| \overline{\boldsymbol{\omega}}\right)+\nabla \pi=\mathbf{f} \\
\operatorname{div} \overline{\mathbf{v}}=0
\end{array}\right.
$$

for some modified pressure term $\pi$.

In this paper we show:

1. How to derive systems (1.4) and (1.5) from a standard turbulence modeling procedure,

2. Existence and uniqueness results of classes of weak solutions for these systems supplemented with smooth enough initial data and Dirichlet homogeneous boundary conditions, under certain reasonable mathematical assumptions.

The main mathematical result we prove is the following.

Theorem 1.1. Assume that the domain $\Omega$ is bounded and smooth (not necessarily with a flat boundary) and that the function $\ell: \Omega \rightarrow \mathbb{R}^{+}$is of class $C^{2}$ and satisfies the two following properties:

$$
\ell(\mathbf{x}) \approx \sqrt{d(\mathbf{x}, \partial \Omega)} \quad \text { for } \mathbf{x} \text { close to } \partial \Omega,
$$

where $d(\mathbf{x}, \partial \Omega)$ denotes the distance from the boundary, and

$$
\forall K \subset \subset \Omega, \exists \ell_{K} \in \mathbb{R}_{+}^{\star} \quad \text { s.t. } \quad \ell(\mathbf{x}) \geq \ell_{K}>0 \quad \forall \mathbf{x} \in K .
$$

Assume in addition that $\mathbf{f} \in L^{2}\left(0, T ; L^{2}(\Omega)^{3}\right)$ and ${ }^{2} \overline{\mathbf{v}}_{0} \in W_{0, \sigma}^{1,3}(\Omega)$. Then, system (1.5) with $\overline{\mathbf{v}}(0)=\overline{\mathbf{v}}_{0}$ in $\Omega$ and $\overline{\mathbf{v}}=\mathbf{0}$ on $(0, T) \times \partial \Omega$ has a unique "regular weak" solution.

\footnotetext{
${ }^{2}$ The divergence-free spaces $W_{0, \sigma}^{1, p}(\Omega)$ are defined below by $(2.5)$.
} 
Theorem 1.1 is a consequence of the weighted estimate (5.1) below, which is the main mathematical result of this paper and of a proper application of monotonicity techniques, coupled with localization of the test functions.

Plan of the paper. The paper is organized as follows: In Section 2 we set the mathematical framework that we use in the whole paper. Sections 3 and 4 are devoted to modeling and to explain the motivations for the systems (1.4) and (1.5). The proof of the main weighted estimate (5.1) is provided in Section 5. Finally, in Section 6 we present the proof of Theorem 1.1.

\section{Acknowledgments}

The research of LCB that led to the present paper was partially supported by a grant of the group GNAMPA of INdAM and by the project of the University of Pisa within the grant PRA_2018_52 UNIPI Energy and regularity: New techniques for classical PDE problems.

LCB thanks also Prof. Tatsien Li for the kind invitation to the conference "China-Italy Conference on PDEs and Their Applications" (Fudan Univ. Shanghai, PRC, Dec 9-13, 2019). The results of the paper has been developed also after discussion originated during the conference.

RL Thanks Prof. W. Layton for pointing out to us the questions raised by the BaldwinLomax model.

\section{Functional setting}

In the sequel $\Omega \subset \mathbb{R}^{3}$ will be a smooth and bounded open set, as usual we write $\mathbf{x}=$ $\left(x_{1}, x_{2}, x_{3}\right)$ for all $\mathbf{x} \in \mathbb{R}^{3}$. In particular, we assume that the boundary $\partial \Omega$ is of class $C^{0,1}$, such that the normal unit vector $\mathbf{n}$ at the boundary is well defined and other relevant properties hold true. We also define the distance $d(\mathbf{x}, A)$ of a point from a closed set $A \subset \mathbb{R}^{3}$ as follows

$$
d(\mathbf{x}, A):=\inf _{\mathbf{y} \in A}|\mathbf{x}-\mathbf{y}|,
$$

and we denote by $d(\mathbf{x})$ the distance of $\mathbf{x}$ from the boundary of the domain $\Omega$

$$
d(\mathbf{x}):=d(\mathbf{x}, \partial \Omega) \quad \forall \mathbf{x} \in \Omega .
$$

For our analysis we will use the customary Lebesgue $\left(L^{p}(\Omega),\|\cdot\|_{p}\right)$ and Sobolev spaces $\left(W^{k, p}(\Omega),\|\cdot\|_{k, p}\right)$ of integer index $k \in \mathbb{N}$ and with $1 \leq p \leq \infty$. The $L^{2}(\Omega)$-norm will be denoted by $\|$.$\| for simplicity. We use boldface for vectors, matrices and tensors. We$ recall that $L_{0}^{p}(\Omega)$ denotes the subspace of $L^{p}(\Omega)$ with zero mean value, while $W_{0}^{1, p}(\Omega)$ is the closure of the smooth and compactly supported functions with respect to the $\|\cdot\|_{1, p}$-norm. As usual we denote $H_{0}^{1}(\Omega)=W_{0}^{1,2}(\Omega)$. In addition, if $\Omega$ is bounded and if $1<p<\infty$, the following two relevant inequalities hold true:

1) the Poincaré inequality

$$
\exists C_{P}(p, \Omega)>0: \quad\|\mathbf{u}\|_{p} \leq C_{P}\|\nabla \mathbf{u}\|_{p} \quad \forall \mathbf{u} \in W_{0}^{1, p}(\Omega)^{3} ;
$$

2) the Korn inequality

$$
\exists C_{K}(p, \Omega)>0: \quad\|\nabla \mathbf{u}\|_{p} \leq C_{K}\|\mathbf{D u}\|_{p} \quad \forall \mathbf{u} \in W_{0}^{1, p}(\Omega)^{3} .
$$


The Korn inequality allows to control the full gradient in $L^{p}(\Omega)$ by its symmetric part, for functions which are zero at the boundary (cf. Malek, Nečas, Rokyta, and Ružička [15]). Classical results (cf. Bourguignon and Brezis [5]) concern controlling the full gradient with curl \& divergence. The following inequality holds true: For all $s \geq 1$ and $1<p<\infty$, there exists a constant $C=C(s, p, \Omega)$ such that,

$$
\|\mathbf{u}\|_{s, p} \leq C\left[\|\operatorname{div} \mathbf{u}\|_{s-1, p}+\|\operatorname{curl} \mathbf{u}\|_{s-1, p}+\|\mathbf{u} \cdot \mathbf{n}\|_{s-1 / p, p, \partial \Omega}+\|\mathbf{u}\|_{s-1, p}\right],
$$

for all $\mathbf{u} \in W^{s, p}(\Omega)^{3}$, where $\|\cdot\|_{s-1 / p, p, \partial \Omega}$ is the trace norm as explained below. This same result has been later improved by von Wahl [21] obtaining, under geometric conditions on the domain, the following estimate without lower order terms: Let $\Omega$ be such that $b_{1}(\Omega)=b_{2}(\Omega)=0$, where $b_{i}(\Omega)$ denotes the i-th Betti number, that is the the dimension of the i-th homology group $H^{i}(\Omega, \mathbb{Z})$. Then, there exists $C=C(p, \Omega)$ such that

$$
\|\nabla \mathbf{u}\|_{p} \leq C\left(\|\operatorname{div} \mathbf{u}\|_{p}+\|\operatorname{curl} \mathbf{u}\|_{p}\right),
$$

for all $\mathbf{u} \in W^{1, p}(\Omega)^{3}$ satisfying either $(\mathbf{u} \cdot \mathbf{n})_{\mid \partial \Omega}=0$ or $(\mathbf{u} \times \mathbf{n})_{\mid \partial \Omega}=\mathbf{0}$. As usual in fluid mechanics, when working with incompressible fluids, it is natural to incorporate the divergence-free constraint directly in the function spaces. These spaces are built upon completing the space of solenoidal smooth vector fields with compact support, denoted as $\phi \in C_{0, \sigma}^{\infty}(\Omega)^{3}$, in an appropriate topology. For $1<p<\infty$ we define

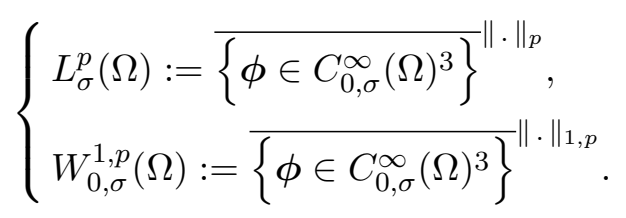

A basic tool in mathematical fluid mechanics is the construction of a continuous right inverse of the divergence operator with zero Dirichlet boundary conditions. An explicit construction is due to the Bogovskiı̌ and it is reviewed in Galdi [8, Ch. 3]. The following results holds true.

Proposition 2.1. Let $\omega \subset \mathbb{R}^{3}$ be a bounded smooth domain and let $f \in L_{0}^{p}(\omega)$. Then, there exists at least one $\mathbf{u}=\operatorname{Bog}_{\omega}(f) \in W_{0}^{1, p}(\omega)^{3}$ which solves the boundary value problem

$$
\left\{\begin{array}{rr}
\operatorname{div} \mathbf{u}=f & \text { in } \omega, \\
\mathbf{u}=\mathbf{0} & \text { on } \partial \omega .
\end{array}\right.
$$

Among other spaces, the operator $\operatorname{Bog}_{\omega}$ is linear and continuous from $L_{0}^{p}(\omega)$ to $W_{0}^{1, p}(\omega)^{3}$, for all $p \in(1, \infty)$.

\section{Part I}

\section{Modeling}

In this part we perform the modeling leading to the model (1.4) in Section 3 and the rotational back-scatter model (1.5) in Section 4. 


\section{On the Baldwin \& Lomax model}

We start by recalling some facts about the Baldwin \& Lomax model which will be used later on. Let $\Omega \subset \mathbb{R}^{3}$ denote the flow domain. We decompose any field $\psi=\psi(t, \mathbf{x})$ with $(t, \mathbf{x}) \in \mathbb{R}_{+} \times \Omega$, as the sum of its mean (denoted by a bar) and its fluctuation,

$$
\psi=\bar{\psi}+\psi^{\prime}
$$

as suggested by Reynolds [4, 7]. The bar operator denotes any linear statistical filter that does not need to be specified, beside that we assume it verifies at least the Reynolds rules:

$$
\partial \bar{\psi}=\overline{\partial \psi} \quad \text { and } \quad \overline{\bar{\psi}}=\bar{\psi},
$$

for any linear differential operator $\partial$.

Let us start by considering the following rotational form of the Navier-Stokes equations (NSE in the sequel),

$$
\left\{\begin{array}{r}
\mathbf{v}_{t}+\boldsymbol{\omega} \times \mathbf{v}-\nu \Delta \mathbf{v}+\nabla\left(p+\frac{|\mathbf{v}|^{2}}{2}\right)=\mathbf{f}, \\
\operatorname{div} \mathbf{v}=0,
\end{array}\right.
$$

where $(\mathbf{v}, p)$ denotes the pair of the velocity and the pressure, and the alternative form of the convective term follows by using the well-known identity

$$
(\mathbf{v} \cdot \nabla) \mathbf{v}=\frac{1}{2} \nabla|\mathbf{v}|^{2}+\boldsymbol{\omega} \times \mathbf{v}
$$

where $\boldsymbol{\omega}=$ curl $\mathbf{v}$. We apply the bar operator to (3.2). By using the Reynolds rules (3.1), one obtains to the following system

$$
\left\{\begin{array}{r}
\overline{\mathbf{v}}_{t}+\overline{\boldsymbol{\omega}} \times \overline{\mathbf{v}}+\overline{\boldsymbol{\omega}^{\prime} \times \mathbf{v}^{\prime}}-\nu \Delta \overline{\mathbf{v}}+\nabla \bar{q}=\mathbf{f}, \\
\operatorname{div} \overline{\mathbf{v}}=0,
\end{array}\right.
$$

where the force is chosen such that $\mathbf{f}=\overline{\mathbf{f}}$ for simplicity. The Bernoulli pressure and the fluctuation of the vorticity are given, respectively, by

$$
q=p+\frac{|\mathbf{v}|^{2}}{2} \quad \text { and } \quad \boldsymbol{\omega}^{\prime}=\operatorname{curl} \mathbf{v}^{\prime}
$$

This leads to the issue of modeling the turbulent flux term $\overline{\boldsymbol{\omega}^{\prime} \times \mathbf{v}^{\prime}}$ only by mean (averaged) quantities. According to the Helmholtz-Hodge theorem, under reasonable regularity and decay assumptions, there exists a unique vector field $\mathbf{A}^{(\mathrm{R})}$ such that

$$
\left\{\begin{aligned}
\operatorname{curl} \mathbf{A}^{(\mathrm{R})} & =\overline{\boldsymbol{\omega}^{\prime} \times \mathbf{v}^{\prime}}, \\
\operatorname{div} \mathbf{A}^{(\mathrm{R})} & =0,
\end{aligned}\right.
$$

and in what follows we call $\mathbf{A}^{(\mathrm{R})}$ the "rotational Reynolds stress." As usual in turbulent modeling, the fundamental question is how to express $\mathbf{A}^{(\mathrm{R})}$ in terms of averaged quantities. It is natural to assume that $\mathbf{A}^{(\mathrm{R})}$ is a function of the mean vorticity $\overline{\boldsymbol{\omega}}$. Following the standard Reynolds-stress modeling-procedure and respecting the divergence free constraint $\operatorname{div} \mathbf{A}^{(\mathrm{R})}=0$, we are led to set

$$
\mathbf{A}^{(\mathrm{R})}=\nu_{\mathrm{turb}} \overline{\boldsymbol{\omega}}+\nabla \psi,
$$


for some scalar function $\psi$ which will be specified later on. Notice that from the Reynolds rules combined with the Schwarz theorem, we have $\operatorname{div} \overline{\boldsymbol{\omega}}=0$. Therefore, taking the divergence of (3.5) and using $\operatorname{div} \mathbf{A}^{(\mathrm{R})}=0$ yields a Poisson equation for $\psi$ :

$$
-\Delta \psi=\operatorname{div}\left(\nu_{\mathrm{turb}} \overline{\boldsymbol{\omega}}\right),
$$

hence, since $\overline{\boldsymbol{\omega}}$ is divergence-free,

$$
\psi=(-\Delta)^{-1}\left(\nabla \nu_{\text {turb }} \cdot \bar{\omega}\right) .
$$

In conclusion, the closure assumption for the rotational Reynolds stress can be expressed as follows

$$
\mathbf{A}^{(\mathrm{R})}=\nu_{\mathrm{turb}} \overline{\boldsymbol{\omega}}+\nabla(-\Delta)^{-1}\left(\nabla \nu_{\mathrm{turb}} \cdot \overline{\boldsymbol{\omega}}\right)
$$

Taking the curl of (3.6) gives

$$
\operatorname{curl} \mathbf{A}^{(\mathrm{R})}=\operatorname{curl}\left(\nu_{\text {turb }} \overline{\boldsymbol{\omega}}\right) .
$$

Therefore, according to the Baldwin \& Lomax model if $\nu_{\text {turb }}=\kappa \ell^{2}(\mathbf{x})|\overline{\boldsymbol{\omega}}|$, and by noting that

$$
\left\{\begin{aligned}
\frac{1}{2} \overline{|\mathbf{v}|^{2}} & =\frac{1}{2}|\overline{\mathbf{v}}|^{2}+k, \\
\operatorname{div}(\overline{\mathbf{v}} \otimes \overline{\mathbf{v}}) & =\overline{\boldsymbol{\omega}} \times \overline{\mathbf{v}}+\nabla\left(\frac{|\overline{\mathbf{v}}|^{2}}{2}\right),
\end{aligned}\right.
$$

(where $k=\frac{1}{2} \overline{\left.\mathbf{v}^{\prime}\right|^{2}}$ denotes the turbulent kinetic energy) we get as closure equations from (3.3) the following system:

$$
\left\{\begin{array}{r}
\overline{\mathbf{v}}_{t}+\operatorname{div}(\overline{\mathbf{v}} \otimes \overline{\mathbf{v}})-\nu \Delta \overline{\mathbf{v}}+\operatorname{curl}\left(\kappa \ell^{2}(\mathbf{x})|\overline{\boldsymbol{\omega}}| \overline{\boldsymbol{\omega}}\right)+\nabla(\bar{p}+k)=\mathbf{f}, \\
\operatorname{div} \overline{\mathbf{v}}=0,
\end{array}\right.
$$

which yields to the system (1.4) by setting the modified pressure $\pi=\bar{p}+k$, and where we recall that $\overline{\boldsymbol{\omega}}=\operatorname{curl} \overline{\mathbf{v}}$. In a vorticity/velocity formulation it is also relevant to consider the rotational form of the convective term, hence

$$
\left\{\begin{array}{r}
\overline{\mathbf{v}}_{t}+\overline{\boldsymbol{\omega}} \times \overline{\mathbf{v}}-\nu \Delta \overline{\mathbf{v}}+\operatorname{curl}\left(\kappa \ell^{2}(\mathbf{x})|\overline{\boldsymbol{\omega}}| \overline{\boldsymbol{\omega}}\right)+\nabla \bar{q}=\mathbf{f} \\
\operatorname{div} \overline{\mathbf{v}}=0 .
\end{array}\right.
$$

\section{Introduction of the rotational back-scatter term}

Following a modeling similar to that already employed in [1], we show in this section how to derive the following model

$$
\left\{\begin{array}{r}
\overline{\mathbf{v}}_{t}+\operatorname{curl}\left(\ell^{2} \overline{\boldsymbol{\omega}}_{t}\right)+\overline{\boldsymbol{\omega}} \times \overline{\mathbf{v}}-\nu \Delta \overline{\mathbf{v}}+\operatorname{curl}\left(\kappa \ell^{2}|\overline{\boldsymbol{\omega}}| \overline{\boldsymbol{\omega}}\right)+\nabla \bar{q}=\mathbf{f}, \\
\operatorname{div} \overline{\mathbf{v}}=0,
\end{array}\right.
$$

for a turbulent flow evolving towards a statistical equilibrium.

Equation (3.3) combined with (3.4) becomes:

$$
\left\{\begin{array}{r}
\overline{\mathbf{v}}_{t}+\overline{\boldsymbol{\omega}} \times \overline{\mathbf{v}}-\nu \Delta \overline{\mathbf{v}}+\operatorname{curl} \mathbf{A}^{(\mathrm{R})}+\nabla \bar{q}=\mathbf{f}, \\
\operatorname{div} \overline{\mathbf{v}}=0 .
\end{array}\right.
$$


According to Leray's result [13], we know any turbulent solution (smooth enough to carry on all the calculations) to (4.2) satisfies the energy inequality

$$
\frac{1}{2} \frac{d}{d t}\|\overline{\mathbf{v}}(t)\|^{2}+\nu\|\nabla \overline{\mathbf{v}}(t)\|^{2}+\left\langle\operatorname{curl} \mathbf{A}^{(\mathrm{R})}, \overline{\mathbf{v}}(t)\right\rangle \leq\langle\mathbf{f}(t), \overline{\mathbf{v}}(t)\rangle,
$$

in the sense of distributions over $(0, T)$, provided that the boundary conditions do not bring additional terms (such as occurs i) with the no-slip boundary condition; ii) when $\Omega=\mathbb{R}^{3}$, or iii) in the space periodic case, for instance). Let us set

$$
\mathscr{I}(t):=\left\langle\operatorname{curl} \mathbf{A}^{(\mathrm{R})}, \overline{\mathbf{v}}(t)\right\rangle .
$$

The aim of what follows is to study the contribution of this term in the energy inequality (4.3). To do so, we use the well-known formula

$$
\nu_{\text {turb }}=C_{k} \ell \sqrt{k},
$$

relating eddy viscosity $\nu_{\text {turb }}$ and turbulent kinetic energy $k$, see [7]. Then, we combine (4.4) with $\nu_{\text {turb }}=\kappa \ell^{2}|\overline{\boldsymbol{\omega}}|$, leading to the closure equation for $k$

$$
k=\frac{\ell^{2}}{2}|\overline{\boldsymbol{\omega}}|^{2}=\frac{\ell^{2}}{2}|\operatorname{curl} \overline{\mathbf{v}}|^{2} .
$$

We assume now that the production of turbulent kinetic energy is mainly due to small scales eddies, which are in a statistical equilibrium and that no-stratification occurs. By a straightforward generalization of what is done in [7, Sec. 4.4.1], we get the following equation for $k$ :

$$
k_{t}+\overline{\mathbf{v}} \cdot \nabla k+\operatorname{div}\left(\overline{e^{\prime} \mathbf{v}^{\prime}}\right)=\mathbf{A}^{(\mathrm{R})} \cdot \overline{\boldsymbol{\omega}}-\varepsilon+\overline{\mathbf{f}^{\prime} \cdot \mathbf{v}^{\prime}},
$$

where the rotational turbulent dissipation is given in this case by $\varepsilon=\nu \overline{\left|\boldsymbol{\omega}^{\prime}\right|^{2}}$, and $e^{\prime}$ denotes the fluctuation of the kinetic energy of the fluctuation $e=\frac{1}{2}\left|\mathbf{v}^{\prime}\right|^{2}$. The combination of (4.5) and (4.6) gives the formal following energy equality:

$$
\frac{d}{d t} \int_{\Omega} k(t)=\int_{\Omega} \ell^{2} \overline{\boldsymbol{\omega}}_{t} \cdot \overline{\boldsymbol{\omega}}=\mathscr{I}(t)-\int_{\Omega} \varepsilon(t)+\overline{\left\langle\mathbf{f}^{\prime}, \mathbf{v}^{\prime}\right\rangle} .
$$

From (4.3) and (4.7) it follows the following inequality

$$
\operatorname{frac} 12 \frac{d}{d t}\left(\|\overline{\mathbf{v}}(t)\|^{2}+\|\ell \overline{\boldsymbol{\omega}}(t)\|^{2}\right)+\nu\|\nabla \overline{\mathbf{v}}(t)\|^{2}+\|\sqrt{\varepsilon}(t)\|^{2} \leq\langle\mathbf{f}(t), \overline{\mathbf{v}}(t)\rangle+\overline{\left\langle\mathbf{f}^{\prime}, \mathbf{v}^{\prime}\right\rangle} .
$$

The energy inequality (4.8) suggests to add the term $\ell^{2} \overline{\boldsymbol{\omega}}_{t}$ to the rotational Reynolds stress in formula (3.6), leading to the following expression for the no-equilibrium rotational Reynolds stress

$$
\mathbf{A}^{(\mathrm{R})}=\ell^{2} \overline{\boldsymbol{\omega}}_{t}+\nu_{\text {turb }} \overline{\boldsymbol{\omega}}+\nabla(-\Delta)^{-1}\left(\nabla \nu_{\text {turb }} \cdot \overline{\boldsymbol{\omega}}\right) .
$$

When we plug (4.9) into (4.2) to get the following energy inequality

$$
\frac{1}{2} \frac{d}{d t}\left(\|\overline{\mathbf{v}}(t)\|^{2}+\|\ell \overline{\boldsymbol{\omega}}(t)\|^{2}\right)+\nu\|\nabla \overline{\mathbf{v}}(t)\|^{2}+\left\|\sqrt{\nu_{\text {turb }}} \overline{\boldsymbol{\omega}}(t)\right\|^{2} \leq\langle\mathbf{f}(t), \overline{\mathbf{v}}(t)\rangle .
$$

We compare (4.8) and (4.10), which is consistent when the following compatibility condition is satisfied:

$$
\left\|\sqrt{\nu_{\text {turb }}} \overline{\boldsymbol{\omega}}(t)\right\|^{2}+\overline{\left\langle\mathbf{f}^{\prime}, \mathbf{v}^{\prime}\right\rangle} \leq\|\sqrt{\varepsilon}(t)\|^{2},
$$

which we assume to be held near statistical equilibium. Hence, (4.1) follows by combining (4.2) and (4.9). Finally, (4.1) yields the model (1.5) by setting the modified pressure $\pi=\bar{p}+k$. An example which satisfies (4.11) is given by the following remark. 
Remark 4.1. The assumption in condition (4.11) can be justified as in [1, Remark 2.2]. More precisely, (for a time averaging filter) this condition holds true when source term is constant $\mathbf{f}(t, \mathbf{x})=\mathbf{f}(\mathbf{x})$, without turbulent fluctuation, i.e., $\mathbf{f}^{\prime}=\mathbf{0}$. It implies a decrease of TKE, which means a decrease of the turbulence, towards a laminar state, or a stable statistical equilibrium, such as a grid turbulence.

\section{Part II}

\section{Analysis of the model}

In this part of the paper we perform the mathematical analysis of the back-scatter rotational model, by using established methods of analysis for non-Newtonian fluids. We first present the main weighted estimate in Section 5 and then proving the existence and uniqueness results in Section 6, as stated in Theorem 1.1.

\section{Main estimate}

In this section we show a bound involving the weighted-curl and weighted-gradient, which does not follow directly from the classical tools combining weighted estimates and harmonic analysis. As employed in [3] it can be shown that for fields in $W_{0, \sigma}^{1, p}(\Omega)$ one can prove the weighted estimate

$$
\int_{\Omega}|\nabla \mathbf{v}(\mathbf{x})|^{p} w(\mathbf{x}) \mathrm{d} \mathbf{x} \leq C(w, \Omega, p) \int_{\Omega}|\operatorname{curl} \mathbf{v}(\mathbf{x})|^{p} w(\mathbf{x}) \mathrm{d} \mathbf{x},
$$

provided that the weight function $w \in L_{l o c}^{1}\left(\mathbb{R}^{3}\right)$, which is s.t. $w \geq 0$ a.e., belongs to the Muckenhoupt class $A_{p}$, for $1<p<\infty$, that is there exists $C$ such that

$$
\sup _{Q \subset \mathbb{R}^{n}}\left(f_{Q} w(\mathbf{x}) \mathrm{d} \mathbf{x}\right)\left(f_{Q} w(\mathbf{x})^{1 /(1-p)} \mathrm{d} \mathbf{x}\right)^{p-1} \leq C,
$$

where $Q$ denotes a cube in $\mathbb{R}^{3}$. It is well-known that the powers of the distance function $w(\mathbf{x})=(d(\mathbf{x}, \partial \Omega))^{\alpha}$ are Muckenhoupt weights of class $A_{p}$ if and only if $-1<\alpha<p-1$, hence in the relevant cases we could not infer the required estimates if $\ell(\mathbf{x})=(d(\mathbf{x}, \partial \Omega))^{\alpha}$, for $\alpha \geq p-1$.

In our case, we can prove a crucial estimate in a different and direct way, by using the special Hilbert structure when $p=2$. Despite being based on elementary direct computations the following lemma plays a fundamental role on the analysis of the rotational back-scatter system (1.5).

Lemma 5.1. Assume that the function $\ell$ is such that $\ell^{2} \in W^{2, \infty}(\Omega)$ and let $\mathbf{v} \in W_{0, \sigma}^{1,2}(\Omega)$. Then, there exists a positive constant $C(\ell)=C\left(\left\|D^{2} \ell^{2}\right\|_{\infty}\right)$ such that

$$
\int_{\Omega} \ell^{2}|\nabla \mathbf{v}|^{2} \mathrm{~d} \mathbf{x} \leq \int_{\Omega} \ell^{2}|\operatorname{curl} \mathbf{v}|^{2} \mathrm{~d} \mathbf{x}+C(\ell) \int_{\Omega}|\mathbf{v}|^{2} \mathrm{~d} \mathbf{x} .
$$

Proof. We start from the well-known vector-calculus identity

$$
-\Delta \mathbf{v}=\operatorname{curl}(\operatorname{curl} \mathbf{v})-\nabla(\operatorname{div} \mathbf{v})=\operatorname{curl}(\operatorname{curl} \mathbf{v})
$$


that holds for any divergence free vector field $\mathbf{v}$. Then multiplying (5.2) by $\ell^{2} \mathbf{v}$ and integrating by parts on $\Omega$ we obtain ${ }^{3}$ :

$$
\int_{\Omega} \nabla \mathbf{v}: \nabla\left(\ell^{2} \mathbf{v}\right) d \mathbf{x}=\int_{\Omega}(\operatorname{curl} \mathbf{v}) \cdot\left(\operatorname{curl}\left(\ell^{2} \mathbf{v}\right)\right) \mathrm{d} \mathbf{x}
$$

where the fact that $\mathbf{v}=\mathbf{0}$ on $\partial \Omega$ has been used. We will explicitly rewrite both sides of (5.3). The left-hand side (l.h.s) of (5.3) can be rewritten as follows

$$
\begin{aligned}
\int_{\Omega} \nabla \mathbf{v}: \nabla\left(\ell^{2} \mathbf{v}\right) \mathrm{d} \mathbf{x} & =\int_{\Omega} \sum_{i, j=1}^{3} \partial_{j} v_{i} \partial_{j}\left(\ell^{2} v_{i}\right) \mathrm{d} \mathbf{x} \\
& =\frac{1}{2} \int_{\Omega} \sum_{i, j=1}^{3} \partial_{j} v_{i}^{2} \partial_{j} \ell^{2} \mathrm{~d} \mathbf{x}+\int_{\Omega} \sum_{i, j=1}^{3}\left(\partial_{j} v_{i}\right)^{2} \ell^{2} \mathrm{~d} \mathbf{x} \\
& =\frac{1}{2} \int_{\Omega} \sum_{j=1}^{3} \partial_{j}\left(\sum_{i=1}^{3} v_{i}^{2}\right) \partial_{j} \ell^{2} \mathrm{~d} \mathbf{x}+\int_{\Omega} \ell^{2}|\nabla v|^{2} \mathrm{~d} \mathbf{x} \\
& =\frac{1}{2} \int_{\Omega} \sum_{j=1}^{3} \partial_{j}|\mathbf{v}|^{2} \partial_{j} \ell^{2} \mathrm{~d} \mathbf{x}+\int_{\Omega} \ell^{2}|\nabla \mathbf{v}|^{2} \mathrm{~d} \mathbf{x} \\
& =\frac{1}{2} \int_{\Omega} \nabla|\mathbf{v}|^{2} \cdot \nabla \ell^{2} \mathrm{~d} \mathbf{x}+\int_{\Omega} \ell^{2}|\nabla \mathbf{v}|^{2} \mathrm{~d} \mathbf{x} \\
& =-\frac{1}{2} \int_{\Omega}|\mathbf{v}|^{2} \Delta \ell^{2} \mathrm{~d} \mathbf{x}+\int_{\Omega} \ell^{2}|\nabla \mathbf{v}|^{2} \mathrm{~d} \mathbf{x},
\end{aligned}
$$

where $\mathbf{v}=\left(v_{1}, v_{2}, v_{3}\right)$ and in the last equality in (5.4) we used integration by parts possible again since $\mathbf{v}=\mathbf{0}$ on $\partial \Omega$. The right-hand side (r.h.s) of (5.3) can be rewritten as

$$
\int_{\Omega}(\operatorname{curl} \mathbf{v}) \cdot\left(\operatorname{curl}\left(\ell^{2} \mathbf{v}\right)\right) \mathrm{d} \mathbf{x}=\int_{\Omega} \ell^{2}|\operatorname{curl} \mathbf{v}|^{2} \mathrm{~d} \mathbf{x}+\int_{\Omega}(\operatorname{curl} \mathbf{v}) \cdot\left(\left(\nabla \ell^{2}\right) \times \mathbf{v}\right) \mathrm{d} \mathbf{x},
$$

where the identity $\operatorname{curl}\left(\ell^{2} \mathbf{v}\right)=\ell^{2} \operatorname{curl} \mathbf{v}+\left(\nabla \ell^{2}\right) \times \mathbf{v}$ has been used. Combining (5.4) and (5.5) yields

$$
\int_{\Omega} \ell^{2}|\nabla \mathbf{v}|^{2} \mathrm{~d} \mathbf{x}=\int_{\Omega} \ell^{2}|\operatorname{curl} \mathbf{v}|^{2} \mathrm{~d} \mathbf{x}+\int_{\Omega}(\operatorname{curl} \mathbf{v}) \cdot\left(\left(\nabla \ell^{2}\right) \times \mathbf{v}\right) \mathrm{d} \mathbf{x}+\frac{1}{2} \int_{\Omega}|\mathbf{v}|^{2} \Delta \ell^{2} \mathrm{~d} \mathbf{x}
$$

To conclude we are going to estimate the two last integrals on the r.h.s of (5.6). To handle the vector product we use the properties of the Ricci totally anti-symmetric tensor $\epsilon_{i j k}$ (such that $\left.(\mathbf{a} \times \mathbf{b})_{i}=\sum_{j=1}^{3} \sum_{k=1}^{3} \epsilon_{i j k} a_{j} b_{k}\right)$ and we rewrite in components the integrand as follows

$$
\begin{aligned}
(\operatorname{curl} \mathbf{v}) \cdot\left(\left(\nabla \ell^{2}\right) \times \mathbf{v}\right) & =\sum_{i, j, k, m, p=1}^{3} \epsilon_{i j k}\left(\partial_{j} v_{k}\right) \epsilon_{i m p}\left(\partial_{m} \ell^{2}\right) v_{p} \\
& =\sum_{j, k, m, p=1}^{3}\left(\delta_{j m} \delta_{k p}-\delta_{j p} \delta_{k m}\right)\left(\partial_{j} v_{k}\right)\left(\partial_{m} \ell^{2}\right) v_{p} \\
& =\sum_{j, k, m=1}^{3}\left[\left(\partial_{j} v_{k}\right)\left(\partial_{m} \ell^{2}\right) v_{k}-\left(\partial_{j} v_{k}\right)\left(\partial_{k} \ell^{2}\right) v_{j}\right] \\
& =\frac{1}{2} \sum_{j, m=1}^{3}\left(\partial_{j}|\mathbf{v}|^{2}\right)\left(\partial_{m} \ell^{2}\right)-\sum_{j, k=1}^{3}\left(\partial_{j} v_{k}\right)\left(\partial_{k} \ell^{2}\right) v_{j} .
\end{aligned}
$$

${ }^{3}$ We denote $\mathbf{A}: \mathbf{B}=\sum_{i, j} a_{i j} b_{i j}$ for any two matrices $\mathbf{A}=\left(a_{i j}\right)$ and $\mathbf{B}=\left(b_{i j}\right)$ for $1 \leq i, j \leq 3$. 
Hence, passing to the integral, and recalling that $\mathbf{v}$ vanishes at the boundary and is divergence-free, we can integrate by parts to obtain

$$
\begin{aligned}
\int_{\Omega}(\operatorname{curl} \mathbf{v}) \cdot\left(\left(\nabla \ell^{2}\right) \times \mathbf{v}\right) \mathrm{d} \mathbf{x} & =\frac{1}{2} \sum_{j, m=1}^{3} \int_{\Omega}\left(\partial_{j}|\mathbf{v}|^{2}\right)\left(\partial_{m} \ell^{2}\right) \mathrm{d} \mathbf{x}-\sum_{j, k=1}^{3} \int_{\Omega}\left(\partial_{j} v_{k}\right)\left(\partial_{k} \ell^{2}\right) v_{j} \mathrm{~d} \mathbf{x} \\
& =-\frac{1}{2} \sum_{j, m=1}^{3} \int_{\Omega}|\mathbf{v}|^{2} \partial_{j m} \ell^{2} \mathrm{~d} \mathbf{x}+\sum_{j, k=1}^{3} \int_{\Omega} v_{j} v_{k} \partial_{j k} \ell^{2} \mathrm{~d} \mathbf{x}
\end{aligned}
$$

which leads to

$$
\left|\int_{\Omega}(\operatorname{curl} \mathbf{v}) \cdot\left(\left(\nabla \ell^{2}\right) \times \mathbf{v}\right) \mathrm{d} \mathbf{x}\right| \leq C\left\|D^{2} \ell^{2}\right\|_{\infty}\|\mathbf{v}\|^{2} .
$$

In addition, the other integral on the r.h.s of (5.6) is bounded by

$$
\frac{1}{2} \int_{\Omega}|\mathbf{v}|^{2} \Delta \ell^{2} \mathrm{~d} \mathbf{x} \leq C\left\|D^{2} \ell^{2}\right\|_{\infty}\|\mathbf{v}\|^{2},
$$

which combines to the previous estimate ending the proof.

\section{$6 \quad$ Existence and uniqueness results}

Throughout this section, we assume that assumptions (1.6) and (1.7) in Theorem 1.1 hold, that is $\ell(\mathbf{x})=O(\sqrt{d(\mathbf{x}, \partial \Omega)})$ near the boundary and $\ell$ is strictly positive inside the domain. Moreover, we also assume that $\mathbf{f} \in L^{2}\left(0, T ; L^{2}(\Omega)^{3}\right)$ and $\overline{\mathbf{v}}_{0} \in W_{0, \sigma}^{1,3}(\Omega)$. Finally, recall that $\overline{\boldsymbol{\omega}}=\operatorname{curl} \overline{\mathbf{v}}$. As in $(2.1)$ we write $d(\mathbf{x}, \partial \Omega)=d(\mathbf{x})$.

Without loss of generality, and according to the modeling introduced in [1] motivated by dimensional analysis, we consider now the back-scatter Baldwin \& Lomax model with the following explicit expression for the length $\ell$

$$
\ell(\mathbf{x})=\sqrt{d_{0} d(\mathbf{x})} \quad \text { for some length } d_{0}>0,
$$

which is consistent with assumptions (1.6) and (1.7) and $\ell^{2}(\mathbf{x})=d_{0} d(\mathbf{x}) \in C^{2}(\bar{\Omega})$, if the boundary of the domain $\partial \Omega$ is at least of class $C^{2}$ (cf. the assumptions in Lemma 5.1 and see also Gilbarg and Trudinger [9, Ch. 14]). Consequently, we now study the existence and uniqueness problems for the following model

$$
\left\{\begin{array}{r}
\overline{\mathbf{v}}_{t}+\operatorname{curl}\left(d_{0} d \overline{\boldsymbol{\omega}}_{t}\right)+\overline{\boldsymbol{\omega}} \times \overline{\mathbf{v}}-\nu \Delta \overline{\mathbf{v}}+\operatorname{curl}\left(d_{0} d|\overline{\boldsymbol{\omega}}| \overline{\boldsymbol{\omega}}\right)+\nabla \bar{p}=\mathbf{f}, \\
\operatorname{div} \overline{\mathbf{v}}=0,
\end{array}\right.
$$

where $\bar{p}$ is some modified pressure and, again for simplicity and without loss of generality, we suppose from now on that $d_{0}=1$. Recall that the above system is supplemented by the Dirichlet boundary conditions $\overline{\mathbf{v}}=\mathbf{0}$ on $(0, T) \times \partial \Omega$ and the initial datum $\overline{\mathbf{v}}(0)=\overline{\mathbf{v}}_{0}$ in $\Omega$.

In order to prove existence of weak solutions we observe that the basic a-priori estimate is obtained by testing with $\overline{\mathbf{v}}$ itself and obtaining (after integration by parts, if solutions are smooth to perform all computations) the following energy inequality for all $s \in(0, T)$

$$
\begin{aligned}
\|\overline{\mathbf{v}}(s)\|^{2}+\|\sqrt{d} \overline{\boldsymbol{\omega}}(s)\|^{2}+\nu \int_{0}^{s}\|\nabla \overline{\mathbf{v}}\|^{2} \mathrm{~d} t & +2 \int_{0}^{s} \int_{\Omega} d|\overline{\boldsymbol{\omega}}|^{3} \mathrm{~d} \mathbf{x} \mathrm{d} t \\
& \leq\left\|\overline{\mathbf{v}}_{0}\right\|^{2}+\left\|\sqrt{d} \overline{\boldsymbol{\omega}}_{0}\right\|^{2}+\frac{C}{\nu} \int_{0}^{s} \int_{\Omega}\|\mathbf{f}\|^{2} \mathrm{~d} \mathbf{x} \mathrm{d} t .
\end{aligned}
$$


Here, the vanishing contribution of the rotational convection term has been used (after modifying the pressure) and the dimensionless constant $C$ comes from applying the Poincaré and Young inequalities. It follows by using (2.2) and a natural $\mathbf{f} \in L^{2}\left(0, T ; L^{2}(\Omega)^{3}\right)$ assumption, that

$$
\begin{aligned}
\overline{\mathbf{v}} & \in L^{\infty}\left(0, T ; L^{2}(\Omega)^{3}\right) \cap L^{2}\left(0, T ; H_{0}^{1}(\Omega)^{3}\right), \\
d^{1 / 2} \overline{\boldsymbol{\omega}} & \in L^{\infty}\left(0, T ; L^{2}(\Omega)^{3}\right), \\
d^{1 / 3} \overline{\boldsymbol{\omega}} & \in L^{3}\left(0, T ; L^{3}(\Omega)^{3}\right) .
\end{aligned}
$$

Hence, from one side we have for the mean velocity $\overline{\mathbf{v}}$ the same estimates valid for the Leray-Hopf weak solutions of the Navier-Stokes equations; On the other side we have further estimates on the mean vorticity which are weighted by the distance from the boundary, hence not enough to directly apply standard methods. We observe that both (dispersive/back-scatter and dissipative/eddy viscosity) the additional degenerate terms pose some mathematical difficulties: If in the system (6.2) one would have been given the following smoothing term

$$
\operatorname{curl}(|\bar{\omega}| \bar{\omega})
$$

then the a-priori estimate, and the divergence-free constraint with (2.4) will imply directly that $\overline{\mathbf{v}} \in L^{3}\left(0, T ; W_{0, \sigma}^{1,3}(\Omega)\right)$, allowing us to apply the same tools valid for the Smagorinsky model as in [14]. Here the estimates degenerate at the boundary (being the mean vorticity weighted by the distance function $d$ ) and this prevents from using the solution itself as a legitimate test function.

Next, if the dispersive term would have been given by

$$
\operatorname{curl}\left(\overline{\boldsymbol{\omega}}_{t}\right)=-\Delta \overline{\mathbf{v}}_{t},
$$

(where the equality is valid for divergence-free functions) the same well-known tools valid for the Voigt model can be used as in [6]. We note in particular that in problem (6.2) the presence of this dispersive term does not allow us to prove by comparison the classical regularity in negative spaces for the time derivative $\overline{\mathbf{v}}_{t}$ (as needed by Aubin-Lions type compactness results); In addition it is also not easy to prove from the weak formulation that the solution is weakly continuous in $L^{2}(\Omega)$ as required by the compactness results à la Hopf (or in the refined form of Landes and Mustonen [12]).

Each term poses some questions which can be separately handled, but the combination of the effects of both weighted terms requires to have a precise interplay between some local (in space) estimates on a double approximated system.

For these reasons we first $\epsilon$-regularize the system by a hyper-dissipative term and we then approximate it by a Galerkin procedure. We first pass to the limit in the Galerkin system and then pass to the limit in the smoothed system, by using further regularity on the time derivative which is obtained in a way similar to [1].

\subsection{The approximate system: existence and further regularity}

For simplicity we assume from now on that $\mathbf{f}=\mathbf{0}$, but the introduction of an external force $\mathbf{f} \in L^{2}\left(0, T ; L^{2}(\Omega)^{3}\right)$ can be done with minor changes. Moreover, throughout the section, we assume $\ell=\sqrt{d}$, but observe that assumptions (1.6) and (1.7) would be enough. 
In order to apply the standard Galerkin method and monotonicity, we approximate the system (6.2) by the following one

$$
\left\{\begin{array}{r}
\overline{\mathbf{v}}_{t}^{\epsilon}+\operatorname{curl}\left(d \overline{\boldsymbol{\omega}}_{t}^{\epsilon}\right)+\overline{\boldsymbol{\omega}}^{\epsilon} \times \overline{\mathbf{v}}^{\epsilon}-\nu \Delta \overline{\mathbf{v}}^{\epsilon}+\operatorname{curl}\left(d\left|\overline{\boldsymbol{\omega}}^{\epsilon}\right| \overline{\boldsymbol{\omega}}^{\epsilon}\right)-\epsilon \operatorname{div}\left(\left|\mathbf{D} \overline{\mathbf{v}}^{\epsilon}\right| \mathbf{D} \overline{\mathbf{v}}^{\epsilon}\right)+\nabla \bar{p}^{\epsilon}=\mathbf{0} \\
\operatorname{div} \overline{\mathbf{v}}^{\epsilon}=0
\end{array}\right.
$$

and we study it with homogeneous Dirichlet boundary conditions.

The above system falls within the standard class of monotone problems as those considered in Lions [14] and Ladyžhenskaya [11] in the analysis of the Smagorinsky model. Here, in addition to the standard Smagorinsky model, we have two perturbation terms, which can be easily handled.

We have the following result.

Theorem 6.1. Let be given $\overline{\mathbf{v}}_{0} \in W_{0, \sigma}^{1,3}(\Omega)$, then there exists a unique weak solution $\overline{\mathbf{v}}^{\epsilon}$ to system (6.3) with $\overline{\mathbf{v}}_{0}$ as initial datum and with homogeneous Dirichlet boundary conditions. This means that

$$
\overline{\mathbf{v}}^{\epsilon} \in L^{\infty}\left(0, T ; L_{\sigma}^{2}(\Omega)^{3}\right) \cap L^{3}\left(0, T ; W_{0, \sigma}^{1,3}(\Omega)\right),
$$

is such that

$$
\begin{aligned}
\int_{0}^{T} & \int_{\Omega}\left(\overline{\boldsymbol{\omega}}^{\epsilon} \times \overline{\mathbf{v}}^{\epsilon}\right) \cdot \boldsymbol{\phi}+\nu \nabla \overline{\boldsymbol{\omega}}^{\epsilon}: \nabla \boldsymbol{\phi}+d\left|\overline{\boldsymbol{\omega}}^{\epsilon}\right| \overline{\boldsymbol{\omega}}^{\epsilon} \cdot \operatorname{curl} \boldsymbol{\phi}+\epsilon\left|\mathbf{D} \overline{\mathbf{v}}^{\epsilon}\right| \mathbf{D} \overline{\mathbf{v}}^{\epsilon}: \mathbf{D} \boldsymbol{\phi} \mathrm{d} \mathbf{x} \mathrm{d} t \\
& =\int_{0}^{T} \int_{\Omega} \overline{\mathbf{v}}^{\epsilon} \cdot \boldsymbol{\phi}_{t}+d \overline{\boldsymbol{\omega}}^{\epsilon} \cdot \operatorname{curl} \phi_{t} \mathrm{~d} \mathbf{x} \mathrm{d} t+\int_{\Omega} \overline{\mathbf{v}}_{0} \cdot \boldsymbol{\phi}(0)+d \overline{\boldsymbol{\omega}}_{0} \cdot \operatorname{curl} \boldsymbol{\phi}(0) \mathrm{d} \mathbf{x}
\end{aligned}
$$

for all $\phi \in C_{0, \sigma}^{\infty}([0, T) \times \Omega)^{3}$.

Proof. Testing by divergence free test vector fields as is custom, we do not consider the pressure term that can be recovered through the usual ways. The proof is based on an application of the Galerkin method to prove existence of approximate solutions. Denoting by $\overline{\mathbf{v}}^{\epsilon, m} \in V_{m}$ for all $t \in(0, T)$ a finite dimensional approximation to $\overline{\mathbf{v}}^{\epsilon}$ one has the following energy estimate for all $s \in[0, T]$

$$
\begin{aligned}
\left\|\overline{\mathbf{v}}^{\epsilon, m}(s)\right\|^{2}+\left\|\sqrt{d} \overline{\boldsymbol{\omega}}^{\epsilon, m}(s)\right\|^{2} & +2 \nu \int_{0}^{s}\left\|\nabla \overline{\mathbf{v}}^{\epsilon, m}\right\|^{2} \mathrm{~d} t+2 \epsilon \int_{0}^{s}\left\|\mathbf{D} \overline{\mathbf{v}}^{\epsilon, m}\right\|_{3}^{3} \mathrm{~d} t \\
& +2 \int_{0}^{s} \int_{\Omega} d\left|\overline{\boldsymbol{\omega}}^{\epsilon, m}\right|^{3} \mathrm{~d} \mathbf{x} \mathrm{d} t \leq\left\|\overline{\mathbf{v}}_{0}\right\|^{2}+\left\|\sqrt{d} \overline{\boldsymbol{\omega}}_{0}\right\|^{2},
\end{aligned}
$$

which shows, by using (2.2)-(2.3) that

$$
\overline{\mathbf{v}}^{\epsilon, m} \in L^{\infty}\left(0, T ; L_{\sigma}^{2}(\Omega)^{3}\right) \cap L^{3}\left(0, T ; W_{0, \sigma}^{1,3}(\Omega)\right),
$$

with estimates depending on $\epsilon>0$, but independent of $m \in \mathbb{N}$.

Next, testing with $\overline{\mathbf{v}}_{t}$, we can see that the contribution of the rotational convective term can be estimated as follows:

$$
\begin{aligned}
\left|\int_{\Omega}(\overline{\boldsymbol{\omega}} \times \overline{\mathbf{v}}) \cdot \overline{\mathbf{v}}_{t} \mathrm{~d} \mathbf{x}\right| & \leq\left\|\overline{\mathbf{v}}_{t}\right\|\|\overline{\mathbf{v}}\|_{6}\|\overline{\boldsymbol{\omega}}\|_{3} \\
& \leq\left\|\overline{\mathbf{v}}_{t}\right\|\|\overline{\mathbf{v}}\|_{6}\|\nabla \overline{\mathbf{v}}\|_{3} \\
& \leq C\left\|\overline{\mathbf{v}}_{t}\right\|\|\nabla \overline{\mathbf{v}}\|\|\mathbf{D} \overline{\mathbf{v}}\|_{3} \\
& \leq \frac{1}{2}\left\|\overline{\mathbf{v}}_{t}\right\|^{2}+C\|\nabla \overline{\mathbf{v}}\|^{2}\|\mathbf{D} \overline{\mathbf{v}}\|_{3}^{2},
\end{aligned}
$$


for smooth enough $\overline{\mathbf{v}}$, where we used the Korn inequality (2.3).

By using $\overline{\mathbf{v}}_{t}^{\epsilon, m}$ as test function and the previous estimates (where $\mathbf{v}$ is replaced by $\overline{\mathbf{v}}^{\epsilon, m}$ ) we then obtain the following differential inequality

$$
\begin{aligned}
\frac{1}{2}\left(\left\|\overline{\mathbf{v}}_{t}^{\epsilon, m}(s)\right\|^{2}+\left\|\sqrt{d} \overline{\boldsymbol{\omega}}_{t}^{\epsilon, m}(s)\right\|^{2}\right) & +\frac{d}{d t} \frac{\nu}{2}\left\|\nabla \overline{\mathbf{v}}^{\epsilon, m}\right\|^{2}+\frac{d}{d t} \frac{\epsilon}{3}\left\|\mathbf{D} \overline{\mathbf{v}}^{\epsilon, m}\right\|_{3}^{3} \\
& +\frac{d}{3 d t} \int_{\Omega} d\left|\overline{\boldsymbol{\omega}}^{\epsilon, m}\right|^{3} \mathrm{~d} \mathbf{x} \leq C\left\|\nabla \overline{\mathbf{v}}^{\epsilon, m}\right\|^{2}\left\|\mathbf{D} \overline{\mathbf{v}}^{\epsilon, m}\right\|_{3}^{2}
\end{aligned}
$$

An application of the Gronwall lemma -possible since $\overline{\mathbf{v}}^{\epsilon, m} \in L^{3}\left(0, T ; W_{0, \sigma}^{1,3}(\Omega)\right)$ - shows that

$$
\overline{\mathbf{v}}^{\epsilon, m} \in L^{\infty}\left(0, T ; W_{0, \sigma}^{1,3}(\Omega)\right) \quad \text { and } \quad \overline{\mathbf{v}}_{t}^{\epsilon, m} \in L^{2}\left(0, T ; L^{2}(\Omega)^{3}\right),
$$

again uniformly in $m \in \mathbb{N}$. The above estimates with Aubin-Lions compactness lemma (cf. [14]) are enough to infer that, for each fixed $\epsilon>0$, there exists

$$
\overline{\mathbf{v}}^{\epsilon} \in L^{\infty}\left(0, T ; W_{0, \sigma}^{1,3}(\Omega)\right) \cap H^{1}\left(0, T ; L_{\sigma}^{2}(\Omega)\right),
$$

such that when $m \rightarrow+\infty$

$$
\begin{aligned}
\overline{\mathbf{v}}^{\epsilon, m} \stackrel{*}{\rightarrow} \overline{\mathbf{v}}^{\epsilon} & \text { in } L^{\infty}\left(0, T ; W_{0, \sigma}^{1,3}(\Omega)\right), \\
\overline{\mathbf{v}}^{\epsilon, m} \rightarrow \overline{\mathbf{v}}^{\epsilon} & \text { in } L^{3}\left(0, T ; W_{0, \sigma}^{1,3}(\Omega)\right), \\
\overline{\mathbf{v}}_{t}^{\epsilon, m} \rightarrow \overline{\mathbf{v}}_{t}^{\epsilon} & \text { in } L^{2}\left(0, T ; L_{\sigma}^{2}(\Omega)^{3}\right), \\
\sqrt{d} \overline{\boldsymbol{\omega}}_{t}^{\epsilon, m} \rightarrow \sqrt{d} \overline{\boldsymbol{\omega}}_{t}^{\epsilon} & \text { in } L^{2}\left(0, T ; L_{\sigma}^{2}(\Omega)^{3}\right), \\
\left|\mathbf{D} \overline{\mathbf{v}}^{\epsilon, m}\right| \mathbf{D} \overline{\mathbf{v}}^{\epsilon, m} \rightarrow \chi_{1} & \text { in } L^{3 / 2}\left(0, T ; L^{3 / 2}(\Omega)^{9}\right), \\
\left|\mathbf{D} \overline{\mathbf{v}}^{\epsilon, m}\right| \mathbf{D} \overline{\mathbf{v}}^{\epsilon, m} \stackrel{*}{\rightarrow} \chi_{1} & \text { in } L^{\infty}\left(0, T ; L^{3 / 2}(\Omega)^{9}\right), \\
d^{2 / 3}\left|\overline{\boldsymbol{\omega}}^{\epsilon, m}\right| \overline{\boldsymbol{\omega}}^{\epsilon, m} \rightarrow \chi_{2} & \text { in } L^{3 / 2}\left(0, T ; L^{3 / 2}(\Omega)^{3}\right), \\
d^{2 / 3}\left|\overline{\boldsymbol{\omega}}^{\epsilon, m}\right| \overline{\boldsymbol{\omega}}^{\epsilon, m} \stackrel{*}{\rightarrow} \chi_{2} & \text { in } L^{\infty}\left(0, T ; L^{3 / 2}(\Omega)^{3}\right), \\
\overline{\mathbf{v}}^{\epsilon, m} \rightarrow \overline{\mathbf{v}}^{\epsilon} & \text { in } L^{2}\left(0, T ; L_{\sigma}^{q}(\Omega)^{3}\right) \quad \forall q<\infty .
\end{aligned}
$$

The above convergences are enough to pass to the limit in the approximate equations, except in the monotone terms.

In particular, for the Baldwin \& Lomax term, it follows that

$$
\begin{aligned}
& \int_{0}^{T} \int_{\Omega} d\left|\overline{\boldsymbol{\omega}}^{\epsilon, m}\right| \overline{\boldsymbol{\omega}}^{\epsilon, m} \cdot \operatorname{curl} \boldsymbol{\phi} \mathrm{d} \mathbf{x} \mathrm{d} t \\
& =\int_{0}^{T} \int_{\Omega} d^{2 / 3}\left|\overline{\boldsymbol{\omega}}^{\epsilon, m}\right| \overline{\boldsymbol{\omega}}^{\epsilon, m} \cdot d^{1 / 3} \operatorname{curl} \boldsymbol{\phi} \mathrm{d} \mathbf{x} \mathrm{d} t \stackrel{m \rightarrow+\infty}{\rightarrow} \quad \int_{0}^{T} \int_{\Omega} \chi_{2} \cdot d^{1 / 3} \operatorname{curl} \boldsymbol{\phi} \mathrm{d} \mathbf{x} \mathrm{d} t \\
& =\int_{0}^{T} \int_{\Omega} d^{1 / 3} \chi_{2} \cdot \operatorname{curl} \phi \mathrm{d} \mathbf{x} \mathrm{d} t
\end{aligned}
$$

for all smooth functions $\phi$ with compact support. Hence, one gets (the trick of distributing powers of the distance function on the integrands will be used several times in the sequel)

$$
\begin{aligned}
\int_{0}^{T} & \int_{\Omega}(\overline{\boldsymbol{\omega}} \times \overline{\mathbf{v}}) \cdot \boldsymbol{\phi}+\nu \nabla \overline{\boldsymbol{\omega}}: \nabla \boldsymbol{\phi}+\epsilon \chi_{1}: \mathbf{D} \boldsymbol{\phi}+d^{1 / 3} \chi_{2} \cdot \operatorname{curl} \boldsymbol{\phi} \mathrm{d} \mathbf{x} \mathrm{d} t \\
\quad & \int_{0}^{T} \int_{\Omega} \overline{\mathbf{v}} \cdot \boldsymbol{\phi}_{t}+d \overline{\boldsymbol{\omega}} \cdot \operatorname{curl} \phi_{t} \mathrm{~d} \mathbf{x} \mathrm{d} t+\int_{\Omega} \overline{\mathbf{v}}_{0} \cdot \boldsymbol{\phi}(0)+d \overline{\boldsymbol{\omega}}_{0} \cdot \operatorname{curl} \boldsymbol{\phi}(0) \mathrm{d} \mathbf{x}
\end{aligned}
$$


and for almost all $0 \leq s_{0} \leq s \leq T$ it holds

$$
\begin{array}{r}
\frac{1}{2}\left(\left\|\overline{\mathbf{v}}^{\epsilon}(s)\right\|^{2}+\left\|\sqrt{d} \overline{\boldsymbol{\omega}}^{\epsilon}(s)\right\|^{2}\right)+\int_{s_{0}}^{s}\left[\nu\left\|\nabla \overline{\mathbf{v}}^{\epsilon}(t)\right\|^{2}+\int_{\Omega}\left(\epsilon \chi_{1}: \mathbf{D} \overline{\mathbf{v}}^{\epsilon}+d^{1 / 3} \chi_{2} \cdot \overline{\boldsymbol{\omega}}^{\epsilon}\right) \mathrm{d} \mathbf{x}\right] \mathrm{d} t \\
=\frac{1}{2}\left(\left\|\overline{\mathbf{v}}^{\epsilon}\left(s_{0}\right)\right\|^{2}+\left\|\sqrt{d} \overline{\boldsymbol{\omega}}^{\epsilon}\left(s_{0}\right)\right\|^{2}\right) .
\end{array}
$$

Hence, to show that $\overline{\mathbf{v}}^{\epsilon}$ is a solution to (6.3) one needs to prove that

$$
\chi_{1}=\left|\mathbf{D} \overline{\mathbf{v}}^{\epsilon}\right| \mathbf{D} \overline{\mathbf{v}}^{\epsilon} \quad \text { and } \quad \chi_{2}=d^{2 / 3}\left|\overline{\boldsymbol{\omega}}^{\epsilon}\right| \overline{\boldsymbol{\omega}}^{\epsilon},
$$

at least almost everywhere in $(0, T) \times \Omega$.

This can be proved by using the standard monotonicity argument (Minty-Browder trick) as developed in the time evolution problem in $[11,14]$. The only thing to be verified is that the function

$$
\overline{\mathbf{v}}^{\epsilon, m}-\overline{\mathbf{v}}^{\epsilon},
$$

is a legitimate test function. This follows by the regularity of the time derivative we proved. Hence, the classical argument proceeds as in (cf. [14, p. 207]) showing that the approximate solution $\overline{\mathbf{v}}^{\epsilon}$ satisfies

$$
\begin{gathered}
\epsilon \int_{0}^{s} \int_{\Omega}\left(\chi_{1}-|\mathbf{D} \phi| \mathbf{D} \phi\right):\left(\mathbf{D} \overline{\mathbf{v}}^{\epsilon}-\mathbf{D} \phi\right) \mathrm{d} \mathbf{x} \mathrm{d} t \geq 0, \\
\int_{0}^{s} \int_{\Omega}\left(\chi_{2}-d^{2 / 3}|\operatorname{curl} \phi| \operatorname{curl} \phi\right) \cdot\left(d^{1 / 3} \overline{\boldsymbol{\omega}}^{\epsilon}-d^{1 / 3} \operatorname{curl} \boldsymbol{\phi}\right) \mathrm{d} \mathbf{x} \mathrm{d} t \\
\int_{0}^{s} \int_{\Omega}\left(d^{1 / 3} \chi_{2}|\operatorname{curl} \phi| \operatorname{curl} \phi\right) \cdot\left(\overline{\boldsymbol{\omega}}^{\epsilon}-\operatorname{curl} \boldsymbol{\phi}\right) \mathrm{d} \mathbf{x d} t \geq 0,
\end{gathered}
$$

for a.e. $s \in[0, T]$ and for arbitrary $\phi \in L^{3}\left(0, T ; W_{0, \sigma}^{1,3}(\Omega)\right)$, since they are both coming from monotone terms. This is enough to imply by monotonicity of the functions

$$
\mathbf{B} \mapsto|\mathbf{B}| \mathbf{B} \quad \text { and } \quad \mathbf{b} \mapsto d^{\alpha}|\mathbf{b}| \mathbf{b},
$$

(which is valid for all matrices $\mathbf{B}$, vectors $\mathbf{b}, \alpha \in \mathbb{R}^{+}$, and smooth functions $d$ such that $d>0$ for all $\mathbf{x} \in \Omega$, cf. [3, Lem. 3.2]) that the equalities in (6.5) hold true. We finally proved that there exists $\overline{\mathbf{v}}^{\epsilon}$ such that

$$
\begin{aligned}
& \int_{0}^{T} \int_{\Omega}\left[\overline{\mathbf{v}}_{t}^{\epsilon} \cdot \boldsymbol{\phi}+d \overline{\boldsymbol{\omega}}_{t}^{\epsilon}(s) \cdot \operatorname{curl} \boldsymbol{\phi}+\left(\overline{\boldsymbol{\omega}}^{\epsilon} \times \overline{\mathbf{v}}^{\epsilon}\right) \cdot \boldsymbol{\phi}\right] \mathrm{d} \mathbf{x} \\
& +\int_{0}^{T} \int_{\Omega}\left[\nu \nabla \overline{\mathbf{v}}^{\epsilon}: \nabla \boldsymbol{\phi}+\epsilon\left|\mathbf{D} \overline{\mathbf{v}}^{\epsilon}\right| \mathbf{D} \overline{\mathbf{v}}^{\epsilon}: \mathbf{D} \boldsymbol{\phi}+d\left|\overline{\boldsymbol{\omega}}^{\epsilon}\right| \overline{\boldsymbol{\omega}}^{\epsilon} \cdot \operatorname{curl} \boldsymbol{\phi}\right] \mathrm{d} \mathbf{x} \mathrm{d} t=0,
\end{aligned}
$$

at least for all $\phi \in L^{3}\left(0, T ; W_{0, \sigma}^{1,3}(\Omega)\right)$. Well-known estimates can be also applied to show that the solution $\overline{\mathbf{v}}^{\epsilon}$ is unique.

Remark 6.1. Due to the regularity of the solution of the approximated system we can use the function $\overline{\mathbf{v}}^{\epsilon, m}-\overline{\mathbf{v}}^{\epsilon}$ as test function. In the case of the non-regularized system (6.2) we will see that localization in the space variable is needed and this is not compatible with the finite dimensional Galerkin approximation. 


\subsection{Proof of Theorem 1.1}

We now consider the original problem (without the $\epsilon$-regularization) and give the proof of the main result of the paper.

Proof of Theorem 1.1. The proof is divided into two steps. Let us start with the existence part.

Step 1: Existence part. To construct weak solutions to (6.2) we consider the limit $\epsilon \rightarrow 0$ of solutions to (6.3). By the estimate coming from the energy inequality we also have by using (5.1) the following inequality, for all $s \in(0, T)$

$$
\begin{aligned}
\frac{1}{2}\left\|\overline{\mathbf{v}}^{\epsilon}(s)\right\|^{2}+\min \left\{1, \frac{1}{2 C(\ell)}\right\}\left\|\sqrt{d} \nabla \overline{\mathbf{v}}^{\epsilon}(s)\right\|^{2} & +2 \nu \int_{0}^{s}\left\|\nabla \overline{\mathbf{v}}^{\epsilon}\right\|^{2} \mathrm{~d} t+2 \epsilon \int_{0}^{s}\left\|\mathbf{D} \overline{\mathbf{v}}^{\epsilon}\right\|_{3}^{3} \mathrm{~d} t \\
& +2 \int_{0}^{s} \int_{\Omega} d\left|\overline{\boldsymbol{\omega}}^{\epsilon}\right|^{3} \mathrm{~d} \mathbf{x} \mathrm{d} t \leq\left\|\overline{\mathbf{v}}_{0}\right\|^{2}+\left\|\sqrt{d} \overline{\boldsymbol{\omega}}_{0}\right\|^{2},
\end{aligned}
$$

which shows that

$$
\overline{\mathbf{v}}^{\epsilon} \in L^{\infty}\left(0, T ; L_{\sigma}^{2}(\Omega)^{3}\right) \cap L^{2}\left(0, T ; W_{0, \sigma}^{1,2}(\Omega)\right) \quad \text { and } \quad d^{1 / 3} \overline{\boldsymbol{\omega}} \in L^{3}((0, T) \times \Omega),
$$

with estimates independent of $\epsilon>0$. We now extract further information from the other bound which is independent of $\epsilon$, namely

$$
\sqrt{d} \nabla \overline{\mathbf{v}}^{\epsilon} \in L^{2}\left(0, T ; L^{2}(\Omega)^{9}\right),
$$

coming from the other term on the left-hand side. We use now the inequality

$$
\|\mathbf{v}\|_{H^{1 / 2}(\Omega)^{3}} \leq C\|\sqrt{d} \nabla \mathbf{v}\| \quad \forall \mathbf{v} \in W_{0}^{1,2}(\Omega)^{3},
$$

which is a simplification of that proved in [1, Thm. 3.1] and $H^{1 / 2}(\Omega)$ denotes the famous critical fractional Sobolev space.

Here, we have the full gradient instead of the deformation tensor on the right-hand side and since we are working with the Galerkin approximations we need to verify it at least for functions in $W_{0}^{1,2}(\Omega)$, instead that for general distributions: This is why the estimate is less technical than that in [1]. By using the Sobolev embedding $H^{1 / 2}(\Omega) \subset L^{3}(\Omega)$, valid in three space dimensions, we finally have the following version of a classical Lions and Magenes result

$$
\|\mathbf{v}\|_{3} \leq C\|\mathbf{v}\|_{1 / 2,2} \leq C\|\sqrt{d} \nabla \mathbf{v}\| \quad \forall \mathbf{v} \in W_{0}^{1,2}(\Omega)^{3} .
$$

This is still not enough for our purposes, but we pass at the estimate obtained testing with $\overline{\mathbf{v}}_{t}^{\epsilon}$. We can also write the following estimate, which follows as in [1, Sec. 4]

$$
\begin{aligned}
\left|\int_{\Omega}\left(\overline{\boldsymbol{\omega}}^{\epsilon} \times \overline{\mathbf{v}}^{\epsilon}\right) \cdot \overline{\mathbf{v}}_{t}^{\epsilon} \mathrm{d} \mathbf{x}\right| & \leq\left\|\overline{\mathbf{v}}_{t}^{\epsilon}\right\|_{3}\left\|\overline{\mathbf{v}}^{\epsilon}\right\|_{6}\left\|\overline{\boldsymbol{\omega}}^{\epsilon}\right\| \leq C\left\|\overline{\mathbf{v}}_{t}\right\|_{3}\left\|\nabla \overline{\mathbf{v}}^{\epsilon}\right\|^{2} \\
& \leq \frac{1}{2} \min \left\{1, \frac{1}{2 C(\ell)}\right\}\left\|\sqrt{d} \nabla \overline{\mathbf{v}}_{t}^{\epsilon}\right\|^{2}+C_{1}(\ell)\left\|\nabla \overline{\mathbf{v}}^{\epsilon}\right\|^{4},
\end{aligned}
$$

valid for smooth functions for some $C_{1}(\ell)$. At the level of the Galerkin approximation we can use the above estimate and then the bound is inherited by the limit in $m \rightarrow+\infty$. Hence, by testing by $\overline{\mathbf{v}}_{t}^{\epsilon, m}$ the Galerkin system and by using Lemma 5.1, with the estimation 
on the convective term (6.8), we get (after passing to the limit $m \rightarrow+\infty$ ) the following differential inequality

$$
\begin{aligned}
\frac{1}{2}\left\|\overline{\mathbf{v}}_{t}^{\epsilon}\right\|^{2}+\frac{1}{2} \min \left\{1, \frac{1}{2 C(\ell)}\right\}\left\|\sqrt{d} \nabla \overline{\mathbf{v}}_{t}^{\epsilon}\right\|^{2} & +\frac{d}{d t} \frac{\nu}{2}\left\|\nabla \overline{\mathbf{v}}^{\epsilon}\right\|^{2}+\frac{d}{d t} \frac{\epsilon}{3}\left\|\mathbf{D} \overline{\mathbf{v}}^{\epsilon}\right\|_{3}^{3} \\
& +\frac{d}{3 d t} \int_{\Omega} d\left|\overline{\boldsymbol{\omega}}^{\epsilon}\right|^{3} \mathrm{~d} \mathbf{x} \leq C_{1}(\ell)\left\|\nabla \overline{\mathbf{v}}^{\epsilon}\right\|^{4} .
\end{aligned}
$$

In particular, for all $s \in(0, T)$ it holds

$$
\frac{\nu}{2}\left\|\nabla \overline{\mathbf{v}}^{\epsilon}(s)\right\|^{2} \leq \frac{\nu}{2}\left\|\nabla \overline{\mathbf{v}}_{0}\right\|^{2}+\frac{\epsilon}{3}\left\|\mathbf{D} \overline{\mathbf{v}}_{0}\right\|_{3}^{3}+\frac{1}{3} \int_{\Omega} d\left|\overline{\boldsymbol{\omega}}_{0}\right|^{3} \mathrm{~d} \mathbf{x}+C_{1}(\ell) \int_{0}^{s}\left\|\nabla \overline{\mathbf{v}}^{\epsilon}\right\|^{4} \mathrm{~d} t,
$$

which shows that, by using the Gronwall lemma (see for example [1, Lemma 4.1])

$$
\begin{aligned}
\frac{\nu}{2}\left\|\nabla \overline{\mathbf{v}}^{\epsilon}(s)\right\|^{2} & \leq\left(\frac{\nu}{2}\left\|\nabla \overline{\mathbf{v}}_{0}\right\|^{2}+\frac{\epsilon}{3}\left\|\mathbf{D} \overline{\mathbf{v}}_{0}\right\|_{3}^{3}+\frac{1}{3} \int_{\Omega} d\left|\overline{\boldsymbol{\omega}}_{0}\right|^{3} \mathrm{~d} \mathbf{x}\right) \exp \left\{C_{1}(\ell) \int_{0}^{s}\left\|\nabla \overline{\mathbf{v}}^{\epsilon}\right\|^{2} \mathrm{~d} t\right\} \\
& \leq\left(\frac{\nu}{2}\left\|\nabla \overline{\mathbf{v}}_{0}\right\|^{2}+\frac{1}{3} \int_{\Omega} d\left|\overline{\boldsymbol{\omega}}_{0}\right|^{3} \mathrm{~d} \mathbf{x}\right) \exp \left\{\frac{C_{1}(\ell)}{2 \nu}\left(\left\|\overline{\mathbf{v}}_{0}\right\|^{2}+\left\|\sqrt{d} \overline{\boldsymbol{\omega}}_{0}\right\|^{2}\right)\right\} \\
& =: F\left(\ell, \overline{\mathbf{v}}_{0}\right),
\end{aligned}
$$

where we have used the uniform estimate for $\overline{\mathbf{v}}^{\epsilon}$ in $L^{2}\left(0, T ; W_{0}^{1,2}(\Omega)^{3}\right.$, previously proved. Therefore, from the above differential inequality we get, for all $s \in(0, T)$

$$
\begin{aligned}
& \frac{1}{2} \int_{0}^{s}\left(\left\|\overline{\mathbf{v}}_{t}^{\epsilon}\right\|^{2}+\min \left\{1, \frac{1}{2 C(\ell)}\right\}\left\|\sqrt{d} \nabla \overline{\mathbf{v}}_{t}^{\epsilon}\right\|^{2}\right) \mathrm{d} t+\frac{\nu}{2}\left\|\nabla \overline{\mathbf{v}}^{\epsilon}(s)\right\|^{2}+\frac{1}{3} \int_{\Omega} d\left|\overline{\boldsymbol{\omega}}^{\epsilon}(s)\right|^{3} \mathrm{~d} \mathbf{x} \\
& +\frac{\epsilon}{3}\left\|\mathbf{D} \overline{\mathbf{v}}^{\epsilon}(s)\right\|_{3}^{3} \leq \frac{\nu}{2}\left\|\nabla \overline{\mathbf{v}}_{0}\right\|^{2}+\frac{1}{3} \int_{\Omega} d\left|\overline{\boldsymbol{\omega}}_{0}\right|^{3} \mathrm{~d} \mathbf{x}+C_{1}(\ell) F\left(\ell, \overline{\mathbf{v}}_{0}\right)\left(\left\|\overline{\mathbf{v}}_{0}\right\|^{2}+\left\|\sqrt{d} \overline{\boldsymbol{\omega}}_{0}\right\|^{2}\right),
\end{aligned}
$$

for all $\epsilon>0$. The latter implies in particular that

$$
\left.\overline{\mathbf{v}}_{t}^{\epsilon} \in L^{2}\left(0, T ; L^{3}(\Omega)^{3}\right) \cap H^{1 / 2}(\Omega)^{3}\right) \quad \text { and } \quad \overline{\mathbf{v}}^{\epsilon} \in L^{\infty}\left(0, T ; W_{0, \sigma}^{1,2}(\Omega)\right),
$$

with bounds uniform in $\epsilon>0$. (The validity of the estimates can be justified working again with the Galerkin approximation showing estimates not depending on $m$ in a standard way.) We can now use this information to pass to the limit as $\epsilon \rightarrow 0$.

In particular, by the a priori estimates, and since $d>0$ for all $\mathbf{x} \in \Omega$ observe that we can infer

$$
d_{K} \int_{0}^{T} \int_{K}\left|\overline{\boldsymbol{\omega}}^{\epsilon}\right|^{3} \mathrm{~d} \mathbf{x} \mathrm{d} t \leq \int_{0}^{T} \int_{\Omega} d\left|\overline{\boldsymbol{\omega}}^{\epsilon}\right|^{3} \mathrm{~d} \mathbf{x} \mathrm{d} t,
$$

with $0<d_{K}:=\min _{\mathbf{x} \in K} d(\mathbf{x})$. Next, being the right-hand side bounded independently of $\epsilon>0$ this shows that we have (up to a sub-sequence) $L^{3}$-weak convergence in $(0, T) \times K$. Considering a family of closed balls $\bar{B}_{q, r_{q}} \subset \Omega$ with rational center $q \in \mathbb{Q}^{3}$ and rational radius $r_{q} \in \mathbb{Q}^{+}$which form a covering of $\Omega$, and using a diagonal argument we can show that we can find a sub-sequence $\left\{\overline{\boldsymbol{\omega}}^{\epsilon}\right\}$ converging in $L^{3}$ in any compact set of $(0, T) \times \Omega$. Moreover, one has also the weak-* convergence in $L^{\infty}\left(0, T ; L^{3}(K)\right)$.

By collecting all information coming from the above a priori estimates, we can infer that there exists

$$
\overline{\mathbf{v}} \in W^{1,2}\left(0, T ; L_{\sigma}^{3}(\Omega) \cap H^{1 / 2}(\Omega)^{3}\right) \cap L^{\infty}\left(0, T ; W_{0, \sigma}^{1,2}(\Omega)\right),
$$

with

$$
\overline{\boldsymbol{\omega}} \in L^{\infty}\left(0, T ; L_{l o c}^{3}(\Omega)^{3}\right),
$$


such that

$$
\begin{aligned}
\overline{\mathbf{v}}^{\epsilon} \stackrel{*}{\rightarrow} \overline{\mathbf{v}} & \text { in } L^{\infty}\left(0, T ; W_{0, \sigma}^{1,2}(\Omega)\right), \\
\sqrt{d} \overline{\boldsymbol{\omega}}^{\epsilon} \stackrel{*}{\rightarrow} \sqrt{d} \overline{\boldsymbol{\omega}} & \text { in } L^{\infty}\left(0, T ; L^{2}(\Omega)^{3}\right), \\
\overline{\mathbf{v}}_{t}^{\epsilon} \rightarrow \overline{\mathbf{v}}_{t} & \text { in } L^{2}\left(0, T ; H^{1 / 2}(\Omega)^{3} \cap L_{\sigma}^{3}(\Omega)^{3}\right), \\
\sqrt{d} \overline{\boldsymbol{\omega}}_{t}^{\epsilon} \rightarrow \sqrt{d} \overline{\boldsymbol{\omega}}_{t} & \text { in } L^{2}\left(0, T ; L^{2}(\Omega)^{3}\right), \\
\epsilon\left|\mathbf{D} \overline{\mathbf{v}}^{\epsilon}\right| \mathbf{D} \overline{\mathbf{v}}^{\epsilon} \rightarrow \mathbf{0} & \text { in } L^{3 / 2}\left(0, T ; L^{3 / 2}(\Omega)^{9}\right), \\
d\left|\overline{\boldsymbol{\omega}}^{\epsilon}\right| \overline{\boldsymbol{\omega}}^{\epsilon} \rightarrow \chi & \text { in } L^{3 / 2}\left(0, T ; L^{3 / 2}(\Omega)^{3}\right), \\
d\left|\overline{\boldsymbol{\omega}}^{\epsilon}\right| \overline{\boldsymbol{\omega}}^{\epsilon} \stackrel{*}{\rightarrow} & \text { in } L^{\infty}\left(0, T ; L^{3 / 2}(\Omega)^{3}\right), \\
\overline{\boldsymbol{\omega}}^{\epsilon} \rightarrow \overline{\boldsymbol{\omega}} & \text { in } L^{3}\left(0, T ; L^{3}(K)^{3}\right), \forall K \subset \subset \Omega, \\
\overline{\boldsymbol{\omega}}^{\epsilon} \stackrel{*}{\rightarrow} \overline{\boldsymbol{\omega}} & \text { in } L^{\infty}\left(0, T ; L^{3}(K)^{3}\right), \forall K \subset \subset \Omega,
\end{aligned}
$$

and by Aubin-Lions lemma

$$
\overline{\mathbf{v}}^{\epsilon} \rightarrow \overline{\mathbf{v}}
$$$$
\text { in } L^{2}\left(0, T ; W_{0, \sigma}^{3 / 4,2}(\Omega)^{3}\right) \subset L^{2}\left(0, T ; L^{4}(\Omega)^{3}\right) \text {, }
$$

All terms in the equation with the weak formulation (6.4) for $\overline{\mathbf{v}}^{\epsilon}$ pass to the limit, except the nonlinear one concerning the Baldwin \& Lomax stress tensor. We obtain then

$$
\int_{0}^{T} \int_{\Omega} \overline{\mathbf{v}}_{t} \cdot \boldsymbol{\phi}+d \overline{\boldsymbol{\omega}}_{t} \cdot \operatorname{curl} \boldsymbol{\phi}+(\overline{\boldsymbol{\omega}} \times \overline{\mathbf{v}}) \cdot \boldsymbol{\phi}+\nu \nabla \overline{\mathbf{v}}: \nabla \boldsymbol{\phi}+\chi \cdot \operatorname{curl} \boldsymbol{\phi} \mathrm{d} \mathbf{x} \mathrm{d} t=0,
$$

for all smooth test functions $\phi$ with compact support in $(0, T) \times \Omega$.

The last step is to show that the limit $\overline{\mathbf{v}}$ (and it curl $\overline{\boldsymbol{\omega}}$ ) satisfies the system (6.2) in a weak sense. To this end it would be classical to take the difference between the equation satisfied by $\overline{\mathbf{v}}^{\epsilon}$ and that satisfied by $\overline{\mathbf{v}}$, test by the difference and show that the limit vanishes. This is needed to show that

$$
d\left|\overline{\boldsymbol{\omega}}^{\epsilon}\right| \overline{\boldsymbol{\omega}}^{\epsilon} \rightarrow d|\overline{\boldsymbol{\omega}}| \overline{\boldsymbol{\omega}},
$$

at least a.e. in $(0, T) \times \Omega$. All the other terms work fine, the only problem is then to make sure that the integral below is well-defined

$$
\int_{0}^{T} \int_{\Omega}\left(d\left|\overline{\boldsymbol{\omega}}^{\epsilon}\right| \overline{\boldsymbol{\omega}}^{\epsilon}-d|\overline{\boldsymbol{\omega}}| \overline{\boldsymbol{\omega}}\right) \cdot\left(\overline{\boldsymbol{\omega}}^{\epsilon}-\overline{\boldsymbol{\omega}}\right) \mathrm{d} \mathbf{x} \mathrm{d} t \rightarrow 0,
$$

and to show that it vanishes. The a priori estimates we have on the solution are not enough for this results: the integral in (6.20) can be well-defined if taken over a compact subset of $K \subset \Omega$, being $\overline{\boldsymbol{\omega}} \in L_{\text {loc }}^{3}(\Omega)^{3}$ for a.e. $t \in[0, T]$, but not over the whole domain $\Omega$. In order to overcome this problem we have to localize. So let us fix an open ball $B:=$ $B(\mathbf{x}, R) \subset \Omega$ and take a cut-off function $\left.0 \leq \eta \in C_{0}^{\infty}(\Omega)\right)$ such that

$$
\begin{cases}\eta(x)=1 & \text { if } x \in \overline{B / 2}:=\overline{B(\mathbf{x}, R / 2)}, \\ \eta(x)=0 & \text { if } x \in \Omega \backslash B .\end{cases}
$$

In this way, since for a.e. $t \in(0, T)$ it follows that $\overline{\mathbf{v}}(t) \in L^{3}(\Omega)^{3}$, and $\overline{\boldsymbol{\omega}}(t) \in L^{3}(B)^{3}$ we have that

$$
\begin{aligned}
\eta\left(\overline{\mathbf{v}}^{\epsilon}-\overline{\mathbf{v}}\right)_{\mid \partial B} & =0, \\
\operatorname{div}\left(\eta\left(\overline{\mathbf{v}}^{\epsilon}-\overline{\mathbf{v}}\right)\right) & =\nabla \eta \cdot\left(\overline{\mathbf{v}}^{\epsilon}-\overline{\mathbf{v}}\right) \in L^{3}(B), \\
\operatorname{curl}\left(\eta\left(\overline{\mathbf{v}}^{\epsilon}-\overline{\mathbf{v}}\right)\right) & =\nabla \eta \times\left(\overline{\mathbf{v}}^{\epsilon}-\overline{\mathbf{v}}\right)+\eta\left(\overline{\boldsymbol{\omega}}^{\epsilon}-\overline{\boldsymbol{\omega}}\right) \in L^{3}(B)^{3},
\end{aligned}
$$


it follows then by $(2.4)$ that $\eta\left(\overline{\mathbf{v}}^{\epsilon}-\overline{\mathbf{v}}\right) \in L^{3}\left(0, T ; W_{0}^{1,3}(B)^{3}\right)$. Concerning the regularity, for all $\epsilon>0$ the vector $\eta\left(\overline{\mathbf{v}}^{\epsilon}-\overline{\mathbf{v}}\right)$ will be suitable as test function, but it still not allowed since $\eta\left(\overline{\mathbf{v}}^{\epsilon}-\overline{\mathbf{v}}\right)$ is not divergence-free. So in order to be able to use it we need to subtract its divergence. This can be done by means of the Bogovskiu operator $\operatorname{Bog}_{B}($.$) associated$ to the ball $B$. Note that we are using it for all fixed $t \in[0, T]$ and this does not create problems since the functions are smooth enough to consider the time as a parameter. Hence, a legitimate test function is the following one

$$
\Phi^{\epsilon}:=\left\{\begin{array}{lr}
\eta\left(\overline{\mathbf{v}}^{\epsilon}-\overline{\mathbf{v}}\right)-\operatorname{Bog}_{B}\left(\nabla \eta \cdot\left(\overline{\mathbf{v}}^{\epsilon}-\overline{\mathbf{v}}\right)\right) & \text { in } B, \\
\mathbf{0} & \text { in } \Omega \backslash B .
\end{array}\right.
$$

From the continuity of the Bogovskil operator as in Proposition 2.1 we can infer that $\operatorname{supp} \Phi^{\epsilon} \subset B$ for all $t \in[0, T]$ and

$$
\Phi^{\epsilon} \in L^{\infty}\left(0, T ; W_{0, \sigma}^{1,2}(\Omega)\right) \cap L^{3}\left(0, T ; W_{0, \sigma}^{1,3}(\Omega)\right) .
$$

Moreover, from the convergence of the approximated sequence we also have, by interpolation, that $\overline{\mathbf{v}}^{\epsilon}-\overline{\mathbf{v}} \rightarrow 0$ in $L^{3}\left(0, T ; L^{3}(\Omega)^{3}\right)$, hence

$$
\begin{aligned}
\Phi^{\epsilon} \rightarrow \mathbf{0} & \text { in } L^{3}\left(0, T ; L^{3}(\Omega)^{3}\right), \\
\Phi^{\epsilon} \rightarrow \mathbf{0} & \text { in } L^{3}\left(0, T ; W_{0}^{1,3}(B)^{3}\right), \\
\operatorname{Bog}_{B}\left(\nabla \eta \cdot\left(\overline{\mathbf{v}}^{\epsilon}-\overline{\mathbf{v}}\right)\right) \rightarrow \mathbf{0} & \text { in } L^{3}\left(0, T ; W_{0}^{1,3}(B)^{3}\right) .
\end{aligned}
$$

We then obtain from the weak formulation of the regularized problem (6.6) the following equality

$$
\begin{aligned}
\int_{0}^{T} \int_{\Omega} & \eta\left(d\left|\overline{\boldsymbol{\omega}}^{\epsilon}\right| \overline{\boldsymbol{\omega}}^{\epsilon}-d|\overline{\boldsymbol{\omega}}| \overline{\boldsymbol{\omega}}\right) \cdot\left(\overline{\boldsymbol{\omega}}^{\epsilon}-\overline{\boldsymbol{\omega}}\right) \mathrm{d} \mathbf{x} \mathrm{d} t \\
& =-\int_{0}^{T} \int_{\Omega}\left(d\left|\overline{\boldsymbol{\omega}}^{\epsilon}\right| \overline{\boldsymbol{\omega}}^{\epsilon}-d|\overline{\boldsymbol{\omega}}| \overline{\boldsymbol{\omega}}\right) \cdot \nabla \eta \times\left(\overline{\mathbf{v}}^{m}-\overline{\mathbf{v}}\right) \mathrm{d} \mathbf{x} \mathrm{d} t \\
& +\int_{0}^{T} \int_{\Omega}\left(d\left|\overline{\boldsymbol{\omega}}^{\epsilon}\right| \overline{\boldsymbol{\omega}}^{\epsilon}-d|\overline{\boldsymbol{\omega}}| \overline{\boldsymbol{\omega}}\right) \cdot \operatorname{curl}\left[\operatorname{Bog}_{B}\left(\nabla \eta \cdot\left(\overline{\mathbf{v}}^{m}-\overline{\mathbf{v}}\right)\right)\right] \mathrm{d} \mathbf{x} \mathrm{d} t \\
& -\nu \int_{0}^{T} \int_{\Omega} \mathbf{D}\left(\overline{\mathbf{v}}^{m}-\overline{\mathbf{v}}\right): \mathbf{D} \Phi^{\epsilon} \mathrm{d} \mathbf{x} \mathrm{d} t+\int_{0}^{T} \int_{\Omega}\left(\overline{\boldsymbol{\omega}} \times \overline{\mathbf{v}}-\overline{\boldsymbol{\omega}}^{\epsilon} \times \overline{\mathbf{v}}^{\epsilon}\right) \cdot \Phi^{\epsilon} \mathrm{d} \mathbf{x} \mathrm{d} t \\
& +\int_{0}^{T} \int_{\Omega}\left(d^{1 / 3} \chi-d|\overline{\boldsymbol{\omega}}| \overline{\boldsymbol{\omega}}\right) \cdot \operatorname{curl} \Phi^{\epsilon} \mathrm{d} \mathbf{x} \mathrm{d} t-\epsilon \int_{0}^{T} \int_{\Omega}\left|\mathbf{D} \overline{\mathbf{v}}^{\epsilon}\right| \mathbf{D} \overline{\mathbf{v}}^{\epsilon}: \mathbf{D} \Phi^{\epsilon} \mathrm{d} \mathbf{x} \mathrm{d} t \\
& -\int_{0}^{T} \int_{\Omega}\left(\overline{\mathbf{v}}_{t}^{\epsilon}-\overline{\mathbf{v}}_{t}\right) \cdot \Phi^{\epsilon} \mathrm{d} \mathbf{x} \mathrm{d} t-\int_{0}^{T} \int_{\Omega} d\left(\overline{\boldsymbol{\omega}}_{t}^{\epsilon}-\overline{\boldsymbol{\omega}}_{t}\right) \cdot \operatorname{curl} \Phi^{\epsilon} \mathrm{d} \mathbf{x} \mathrm{d} t \\
& =:(I)+(I I)+(I I I)+(I V)+(V)+(V I)+(V I I)+(V I I I) .
\end{aligned}
$$

The strong $L^{3}\left(0, T ; L^{3}(\Omega)^{3}\right)$ convergence of $\overline{\mathbf{v}}^{m}$ and the continuity of the Bogovskil operator, with (6.21) imply that $(I)$ and $(I I)$ vanish as $\epsilon \rightarrow 0$ (we also used that the function $d$ is uniformly bounded). We write then the following equality

$$
\begin{aligned}
(I I I) & =-\nu \int_{0}^{T} \int_{\Omega} \eta\left|\mathbf{D}\left(\overline{\mathbf{v}}^{m}-\overline{\mathbf{v}}\right)\right|^{2} \mathrm{~d} \mathbf{x} \mathrm{d} t-\nu \int_{0}^{T} \int_{\Omega} \mathbf{D}\left(\overline{\mathbf{v}}^{m}-\overline{\mathbf{v}}\right): \nabla \eta \otimes\left(\overline{\mathbf{v}}^{m}-\overline{\mathbf{v}}\right) \mathrm{d} \mathbf{x} \mathrm{d} t \\
& +\nu \int_{0}^{T} \int_{\Omega} \mathbf{D}\left(\overline{\mathbf{v}}^{m}-\overline{\mathbf{v}}\right): \mathbf{D}\left[\operatorname{Bog}_{B}\left(\nabla \eta \cdot\left(\overline{\mathbf{v}}^{m}-\overline{\mathbf{v}}\right)\right)\right] \mathrm{d} \mathbf{x} \mathrm{d} t
\end{aligned}
$$


where the first term is non-positive and the second and third one vanish on account of (6.21)-(6.23). The convergence of $(I V)$ follows from uniform bounds in $L^{2}\left(0, T ; W^{1,2}(\Omega)^{3}\right)$ and (6.21). The term $(V) \rightarrow 0$ due to $(6.23)$ and the bound in $L^{3 / 2}((0, T) \times B)$ of $\chi$ and $|\overline{\boldsymbol{\omega}}| \overline{\boldsymbol{\omega}}$. Next, $(V I) \rightarrow 0$, due the $L^{3}\left(0, T ; W^{1,3}(B)^{3}\right)$ bound of $\overline{\mathbf{v}}^{m}-\overline{\mathbf{v}}$ and (6.13).

Concerning the terms involving the time derivative, which are the new ones with respect to the steady problem treated in [3], it follows that they both vanish as $\epsilon \rightarrow 0$. In fact, in $(V I I)$ the term $\overline{\mathbf{v}}_{t}^{\epsilon}-\overline{\mathbf{v}}_{t}$ is bounded in $L^{2}\left(0, T ; L^{2}(\Omega)^{3}\right)$, by $(6.19)$, while $\Phi^{\epsilon}$ vanishes strongly in $L^{2}\left(0, T ; L^{2}(\Omega)^{3}\right)$. Moreover, regarding $(V I I I)$, we rewrite it as

$$
\int_{0}^{T} \int_{\Omega}\left(\sqrt{d} \overline{\boldsymbol{\omega}}_{t}^{\epsilon}-\sqrt{d} \overline{\boldsymbol{\omega}}_{t}\right) \cdot \sqrt{d} \Phi^{\epsilon} \mathrm{d} \mathbf{x} \mathrm{d} t
$$

and observe that the quantity $\sqrt{d} \overline{\boldsymbol{\omega}}_{t}^{\epsilon}-\sqrt{d} \overline{\boldsymbol{\omega}}_{t}$ is bounded in $L^{2}\left(0, T ; L^{2}(\Omega)^{3}\right)$ by $(6.12)$, while $\sqrt{d} \Phi^{\epsilon}$ converges strongly to zero in $L^{2}\left(0, T ; L^{2}(\Omega)^{3}\right)$ by (6.18).

In this way we proved that

$$
\begin{aligned}
\min _{\mathbf{x} \in \overline{B / 2}} d(\mathbf{x}) & \int_{0}^{T} \int_{B / 2}\left(\left|\overline{\boldsymbol{\omega}}^{\epsilon}\right| \overline{\boldsymbol{\omega}}^{\epsilon}-|\overline{\boldsymbol{\omega}}| \overline{\boldsymbol{\omega}}\right) \cdot\left(\overline{\boldsymbol{\omega}}^{\epsilon}-\overline{\boldsymbol{\omega}}\right) \mathrm{d} \mathbf{x} \mathrm{d} t \\
& =\min _{\mathbf{x} \in \overline{B / 2}} d(\mathbf{x}) \int_{0}^{T} \int_{B / 2} \eta\left(\left|\overline{\boldsymbol{\omega}}^{\epsilon}\right| \overline{\boldsymbol{\omega}}^{\epsilon}-|\overline{\boldsymbol{\omega}}| \overline{\boldsymbol{\omega}}\right) \cdot\left(\overline{\boldsymbol{\omega}}^{\epsilon}-\overline{\boldsymbol{\omega}}\right) \mathrm{d} \mathbf{x} \mathrm{d} t \\
& \leq \int_{0}^{T} \int_{B / 2} d(\mathbf{x}) \eta\left(\left|\overline{\boldsymbol{\omega}}^{\epsilon}\right| \overline{\boldsymbol{\omega}}^{\epsilon}-|\overline{\boldsymbol{\omega}}| \overline{\boldsymbol{\omega}}\right) \cdot\left(\overline{\boldsymbol{\omega}}^{\epsilon}-\overline{\boldsymbol{\omega}}\right) \mathrm{d} \mathbf{x} \\
& \leq \int_{0}^{T} \int_{B} d(\mathbf{x}) \eta\left(\left|\overline{\boldsymbol{\omega}}^{\epsilon}\right| \overline{\boldsymbol{\omega}}^{\epsilon}-|\overline{\boldsymbol{\omega}}| \overline{\boldsymbol{\omega}}\right) \cdot\left(\overline{\boldsymbol{\omega}}^{\epsilon}-\overline{\boldsymbol{\omega}}\right) \mathrm{d} \mathbf{x} \mathrm{d} t \rightarrow 0
\end{aligned}
$$

which is enough to prove that $\left|\overline{\boldsymbol{\omega}}^{\epsilon}\right| \overline{\boldsymbol{\omega}}^{\epsilon} \rightarrow|\overline{\boldsymbol{\omega}}| \overline{\boldsymbol{\omega}}$ a.e. in $(0, T) \times B / 2$. The arbitrariness of the ball $B \subset \Omega$ implies that

$$
\left|\overline{\boldsymbol{\omega}}^{\epsilon}\right| \overline{\boldsymbol{\omega}}^{\epsilon} \rightarrow|\overline{\boldsymbol{\omega}}| \overline{\boldsymbol{\omega}} \quad \text { a.e. in }(0, T) \times \Omega .
$$

This proves, by the identification of weak and almost everywhere limits, the validity of the limit $d\left|\overline{\boldsymbol{\omega}}^{\epsilon}\right| \overline{\boldsymbol{\omega}}^{\epsilon} \rightarrow d|\overline{\boldsymbol{\omega}}| \overline{\boldsymbol{\omega}}$, at least in $L^{3 / 2}\left(0, T ; L^{3 / 2}(\Omega)^{3}\right)$ ending the proof of the existence part, since $\overline{\mathbf{v}}$ satisfies

$$
\begin{aligned}
& \int_{0}^{T} \int_{\Omega}\left[\overline{\mathbf{v}}_{t} \cdot \boldsymbol{\phi}+d \overline{\boldsymbol{\omega}}_{t} \cdot \operatorname{curl} \boldsymbol{\phi}+(\overline{\boldsymbol{\omega}} \times \overline{\mathbf{v}}) \cdot \boldsymbol{\phi}\right] \mathrm{d} \mathbf{x} \mathrm{d} t \\
& +\int_{0}^{T} \int_{\Omega}[\nu \nabla \overline{\mathbf{v}}: \nabla \boldsymbol{\phi}+d|\overline{\boldsymbol{\omega}}| \overline{\boldsymbol{\omega}} \cdot \operatorname{curl} \boldsymbol{\phi}] \mathrm{d} \mathbf{x} \mathrm{d} t=0,
\end{aligned}
$$

for all $\phi \in C_{0, \sigma}^{\infty}((0, T) \times \Omega)^{3}$.

Observe that the hypotheses on the initial datum $\overline{\mathbf{v}}_{0} \in W_{0, \sigma}^{1,3}(\Omega)$ are enough to make the integrals well-defined. In the limit only the weighted estimate $\int_{\Omega} d\left|\overline{\boldsymbol{\omega}}_{0}\right|^{3} \mathrm{~d} \mathbf{x}<\infty$ is needed. So at the price of further technical questions related to approximation by smooth functions in weighted space as in Kufner [10], one can relax the hypotheses on the initial datum as follows:

$$
\overline{\mathbf{v}}_{0} \in W_{0, \sigma}^{1,2}(\Omega) \quad \text { with } \quad \int_{\Omega} d\left|\overline{\boldsymbol{\omega}}_{0}\right|^{3} \mathrm{~d} \mathbf{x}<\infty
$$

such that there exists a sequence $\overline{\mathbf{v}}_{0}^{\epsilon} \in W_{0, \sigma}^{1,3}(\Omega)$ satisfying

$$
\overline{\mathbf{v}}_{0}^{\epsilon} \rightarrow \overline{\mathbf{v}}_{0} \text { in } W_{0, \sigma}^{1,2}(\Omega) \quad \text { and } \quad \int_{\Omega} d\left|\overline{\boldsymbol{\omega}}_{0}^{\epsilon}\right|^{3} \mathrm{~d} \mathbf{x} \leq 2 \int_{\Omega} d\left|\overline{\boldsymbol{\omega}}_{0}\right|^{3} \mathrm{~d} \mathbf{x} .
$$


We continue now with the uniqueness part.

Step 2: Uniqueness part. Since we proved existence of rather regular weak solutions, we can now prove their uniqueness. As usual we suppose that there exists two solutions $\overline{\mathbf{v}}_{1}, \overline{\mathbf{v}}_{2}$ corresponding to the same initial datum. We take the difference and it follows that all estimates satisfied by the velocity are inherited by the difference and hence $\delta \overline{\mathbf{v}}:=\overline{\mathbf{v}}_{1}-\overline{\mathbf{v}}_{2}$ and $\delta \overline{\boldsymbol{\omega}}:=\overline{\boldsymbol{\omega}}_{1}-\overline{\boldsymbol{\omega}}_{2}$ satisfy in particular the following

$$
\begin{aligned}
\delta \overline{\mathbf{v}} & \in L^{\infty}\left(0, T ; W_{0, \sigma}^{1,2}(\Omega)\right), \\
\sqrt{d}(\delta \overline{\boldsymbol{\omega}}) & \in L^{\infty}\left(0, T ; L^{2}(\Omega)^{3}\right), \\
\delta \overline{\mathbf{v}}_{t} & \in L^{2}\left(0, T ; H^{1 / 2}(\Omega)^{3} \cap L_{\sigma}^{3}(\Omega)\right), \\
\sqrt{d}\left(\delta \overline{\boldsymbol{\omega}}_{t}\right) & \in L^{2}\left(0, T ; L^{2}(\Omega)^{3}\right), \\
\delta \overline{\boldsymbol{\omega}} & \in L^{\infty}\left(0, T ; L^{3}(K)^{3}\right), \quad \forall K \subset \subset \Omega .
\end{aligned}
$$

It follows that if we write the equation satisfied by the difference $\delta \overline{\mathbf{v}}$, we can rigorously test by the difference itself. All terms work directly, the only one that needs to be checked is the monotone one. In fact, if we write

$$
\int_{0}^{T} \int_{\Omega}\left(d\left|\overline{\boldsymbol{\omega}}_{1}\right| \overline{\boldsymbol{\omega}}_{1}-d\left|\overline{\boldsymbol{\omega}}_{2}\right| \overline{\boldsymbol{\omega}}_{2}\right) \cdot\left(\overline{\boldsymbol{\omega}}_{1}-\overline{\boldsymbol{\omega}}_{2}\right) \mathrm{d} \mathbf{x} \mathrm{d} t
$$

this would be surely finite if $\overline{\boldsymbol{\omega}}_{i} \in L^{3}\left(0, T ; L^{3}(\Omega)^{3}\right)$, which we do not know. Nevertheless we can observe that, for all $i, j=1,2$

$$
\left|\int_{0}^{T} \int_{\Omega} d\right| \overline{\boldsymbol{\omega}}_{i}\left|\overline{\boldsymbol{\omega}}_{i} \cdot \overline{\boldsymbol{\omega}}_{j} \mathrm{~d} \mathbf{x} \mathrm{d} t\right| \leq\left(\int_{0}^{T} \int_{\Omega} d\left|\overline{\boldsymbol{\omega}}_{i}\right|^{3} \mathrm{~d} \mathbf{x} \mathrm{d} t\right)^{2 / 3}\left(\int_{0}^{T} \int_{\Omega} d\left|\overline{\boldsymbol{\omega}}_{j}\right|^{3} \mathrm{~d} \mathbf{x} \mathrm{d} t\right)^{1 / 3}<\infty,
$$

hence the integral in (6.24) is well defined, and then by monotonicity it follows that

$$
\int_{0}^{T} \int_{\Omega}\left(d\left|\overline{\boldsymbol{\omega}}_{1}\right| \overline{\boldsymbol{\omega}}_{1}-d\left|\overline{\boldsymbol{\omega}}_{2}\right| \overline{\boldsymbol{\omega}}_{2}\right) \cdot\left(\overline{\boldsymbol{\omega}}_{1}-\overline{\boldsymbol{\omega}}_{2}\right) \mathrm{d} \mathbf{x} \mathrm{d} t \geq 0
$$

This proves that

$$
\frac{1}{2}\|\delta \overline{\mathbf{v}}(s)\|^{2}+\frac{1}{2}\|\sqrt{d}(\delta \overline{\boldsymbol{\omega}}(s))\|^{2}+\frac{\nu}{2} \int_{0}^{s}\|\nabla(\delta \overline{\mathbf{v}})\|^{2} \mathrm{~d} t \leq \frac{C}{\nu} \int_{0}^{s}\left\|\nabla \overline{\mathbf{v}}_{2}\right\|^{4}\|\delta \overline{\mathbf{v}}\|^{2} \mathrm{~d} t,
$$

by using the standard inequalities for the nonlinear term (as in [1, Sec. 4]), since $\delta \overline{\mathbf{v}}(0) \equiv \mathbf{0}$. The bound $\nabla \overline{\mathbf{v}}_{2} \in L^{\infty}\left(0, T ; L^{2}(\Omega)^{9}\right)$ and the Gronwall lemma implies that $\|\delta \overline{\mathbf{v}}(s)\| \equiv 0$ for all $s \in[0, T]$, hence the uniqueness follows.

\section{References}

[1] C. Amrouche, L. C. Berselli, R. Lewandowski, and D. D. Nguyen. Turbulent flows as generalized Kelvin-Voigt materials: Modeling and analysis. Nonlinear Anal., 196:111790, 2020 .

[2] B. Baldwin and H. Lomax. Thin-layer approximation and algebraic model for separated turbulent flows. In 16th Aerospace Sciences Meeting, Huntsville, AL, U.S.A. Aerospace Sciences Meetings, 1978. 
[3] L. C. Berselli and D. Breit. On the existence of weak solutions for the steady BaldwinLomax model and generalizations. Technical Report 2003.00691, arXiv, 2020.

[4] L. C. Berselli, T. Iliescu, and W. J. Layton. Mathematics of Large Eddy Simulation of turbulent flows. Scientific Computation. Springer-Verlag, Berlin, 2006.

[5] J. P. Bourguignon and H. Brezis. Remarks on the Euler equation. J. Functional Analysis, 15:341-363, 1974.

[6] Y. Cao, E. M. Lunasin, and E. S. Titi. Global well-posedness of the three-dimensional viscous and inviscid simplified Bardina turbulence models. Commun. Math. Sci., $4(4): 823-848,2006$.

[7] T. Chacón Rebollo and R. Lewandowski. Mathematical and numerical foundations of turbulence models and applications. Modeling and Simulation in Science, Engineering and Technology. Birkhäuser/Springer, New York, 2014.

[8] G. P. Galdi. An introduction to the mathematical theory of the Navier-Stokes equations. Steady-state problems. Springer Monographs in Mathematics. Springer-Verlag, New York, 2011.

[9] D. Gilbarg and N. S. Trudinger. Elliptic partial differential equations of second order. Classics in Mathematics, Springer-Verlag, Berlin, 2001, Reprint of the 1998 edition.

[10] A. Kufner. Weighted Sobolev spaces. A Wiley-Interscience Publication. John Wiley \& Sons, Inc., New York, 1985. Translated from the Czech.

[11] O. A. Ladyžhenskaya. The mathematical theory of viscous incompressible flow. Second English edition, revised and enlarged. Translated from the Russian by Richard A. Silverman and John Chu. Mathematics and its Applications, Vol. 2. Gordon and Breach Science Publishers, New York, 1969.

[12] R. Landes and V. Mustonen. A strongly nonlinear parabolic initial-boundary value problem. Ark. Mat., 25(1):29-40, 1987.

[13] J. Leray. Sur le mouvement d'un liquide visqueux emplissant l'espace. Acta Math., 63(1):193-248, 1934.

[14] J.-L. Lions. Quelques méthodes de résolution des problèmes aux limites non linéaires. Dunod, Gauthier-Villars, Paris, 1969.

[15] Malek, J., Nečas, J., Rokyta, M., Ružička: Weak and Measure-valued Solutions to Evolutionary PDEs, Chapman \& Hall, London (1996).

[16] A. M. Obuhov. Turbulence in an atmosphere with inhomogeneous temperature. Akad. Nauk SSSR. Trudy Inst. Teoret. Geofiz., 1:95-115, 1946.

[17] L. Prandtl. Prandtl-Essentials of fluid mechanics, volume 158 of Applied Mathematical Sciences. Springer, New York, third edition, 2010. Translated from the 12th German edition by Katherine Asfaw and edited by Herbert Oertel, With contributions by P. Erhard, D. Etling, U. Müller, U. Riedel, K. R. Sreenivasan and J. Warnatz.

[18] Y. Rong, W. Layton, and H. Zhao. Extension of a simplified Baldwin-Lomax model to nonequilibrium turbulence: model, analysis and algorithms. Numer. Methods Partial Differential Equations, 35(5):1821-1846, 2019. 
[19] J. Smagorinsky. On the application of numerical methods to the solution of systems of partial differential equations arising in meteorology. In Frontiers of numerical mathematics, pages 107-125. University of Wisconsin Press, Madison, Wis., 1960.

[20] E. R. van Driest. On turbulent flow near a wall. J. Aerospace Sci., 23:1007-1011, 1956.

[21] W. von Wahl. Estimating $\nabla u$ by $\operatorname{div} u$ and curl $u$. Math. Methods Appl. Sci., 15(2):123-143, 1992. 